\title{
Effective spectral function for quasielastic scattering on nuclei
}

\author{
A. Bodek ${ }^{1, a}$, M. E. Christy ${ }^{2}$, B. Coopersmith ${ }^{1}$ \\ ${ }^{1}$ Department of Physics and Astronomy, University of Rochester, Rochester, NY 14627-0171, USA \\ ${ }^{2}$ Hampton University, Hampton, VA 23668, USA
}

Received: 6 May 2014 / Accepted: 22 September 2014 / Published online: 7 October 2014

(c) The Author(s) 2014. This article is published with open access at Springerlink.com

\begin{abstract}
Spectral functions that are used in neutrino event, generators to model quasielastic $(\mathrm{QE})$ scattering from nuclear targets include Fermi gas, Local Thomas Fermi gas (LTF), Bodek-Ritchie Fermi gas with high momentum tail, and the Benhar-Fantoni two dimensional spectral function. We find that the $v$ dependence of predictions of these spectral functions for the QE differential cross sections $\left(d^{2} \sigma / d Q^{2} d \nu\right)$ are in disagreement with the prediction of the $\psi^{\prime}$ superscaling function which is extracted from fits to quasielastic electron scattering data on nuclear targets. It is known that spectral functions do not fully describe quasielastic scattering because they only model the initial state. Final state interactions distort the shape of the differential cross section at the peak and increase the cross section at the tails of the distribution. We show that the kinematic distributions predicted by the $\psi^{\prime}$ superscaling formalism can be well described with a modified effective spectral function (ESF). By construction, models using ESF in combination with the transverse enhancement contribution correctly predict electron QE scattering data.
\end{abstract}

\section{Introduction}

Neutrino oscillation experiments make use of neutrino Monte Carlo (MC) event generators to model the cross sections and kinematic distributions of the leptonic and hadronic final state of neutrino interactions on nuclear targets. Therefore, reliable simulations of the effects of Fermi motion and other nuclear effects are important. In order to model neutrino cross sections we need to model the vector part, the axial-vector part, and axial-vector interference. Because of the conservation of the vector current (CVC), the same models should be able to reliably predict the QE electron scattering cross section on nuclear targets. Unfortunately, none of the models which are currently implemented in neutrino MC generators are able to

\footnotetext{
a e-mail: bodek@pas.rochester.edu
}

do it. In this paper, we propose an approach which guarantees agreement with quasielaststic (QE) electron scattering data by construction.

\subsection{QE scattering from independent nucleons}

The top panel of Fig. 1 is the general diagram for QE lepton (election, muon or neutrino) scattering from a nucleon which is bound in a nucleus of mass $M_{A}$. In this paper, we focus on charged current neutrino scattering. The scattering is from an off-shell bound neutron of momentum $\mathbf{P}_{\mathbf{i}}=\mathbf{k}$. The onshell recoil $[A-1]^{*}$ (spectator) nucleus has a momentum $\mathbf{P}_{\mathbf{A}-\mathbf{1}}^{*}=\mathbf{P}_{\mathbf{s}}=-\mathbf{k}$. This process is referred to as the $1 \mathrm{p} 1 \mathrm{~h}$ process (one proton one hole). The * is used to indicate that the spectator nucleus is not in the ground state because it has one hole. The four-momentum transfer to the nuclear target is defined as $q=(\mathbf{q}, v)$. Here $v$ is the energy transfer, and $Q^{2}=-q^{2}=v^{2}-\mathbf{q}^{2}$ is the square of the four-momentum transfer. For free nucleons the energy transfer $v$ is equal to $Q^{2} / 2 M_{N}$ where $M_{N}$ is the mass of the nucleon. At a fixed value of $Q^{2}, \mathrm{QE}$ scattering on nucleons bound in a nucleus yields a distribution in $v$ which peaks at $v=Q^{2} / 2 M_{N}$. In this communication, the term "normalized quasielastic distribution" refers to the normalized differential cross section $\frac{1}{\sigma} \frac{d \sigma}{d \nu}\left(Q^{2}, \nu\right)=\frac{d^{2} \sigma / d Q^{2} d v}{<d \sigma / d Q^{2}>}$ where $<\frac{d \sigma}{d Q^{2}}>$ is the integral of $\left[\frac{d^{2} \sigma}{d Q^{2} d \nu}\right] d v$ over all values of $\nu$ (for a given value of $Q^{2}$ ).

The bottom panel of Fig. 1 shows the same QE lepton scattering process, but now also including a final state interaction with another nucleon in the scattering process. This final state interaction modifies the scattering amplitude and therefore can change the kinematics of the final state lepton. In this paper, we refer to it as "final state interaction of the first kind" (FSI).

The final state nucleon can then undergo more interactions with other nucleons in the spectator nucleus. These interactions do not change the energy of the final state lepton. We refer to this kind of final state interaction as "final state inter- 


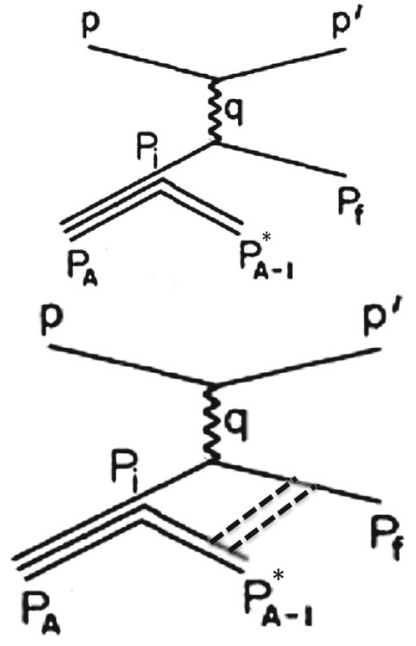

Fig. 1 Top: Scattering from an off-shell bound neutron of momentum $\mathbf{P}_{\mathbf{i}}=\mathbf{k}$ in a nucleus of mass A. The on-shell recoil $[A-1]^{*}$ (spectator) nucleus has a momentum $\mathbf{P}_{\mathbf{A}-\mathbf{1}}^{*}=\mathbf{P}_{\mathbf{s}}=-\mathbf{k}$. This process is referred to as the $1 \mathrm{p} 1 \mathrm{~h}$ process (one proton one hole). Bottom: The $1 \mathrm{p} 1 \mathrm{~h}$ process including final state interaction (of the first kind) with another nucleon

action of the second kind". Final state interactions of the second kind reduce the energy of the final state nucleon.

\subsection{Spectral functions}

In general, neutrino event generators assume that the scattering occurs on independent nucleons which are bound in the nucleus. Generators such as GENIE [1,2], NEUGEN [3], NEUT [4], NUANCE [5] NuWro [6,7] and GiBUU [8] account for nucleon binding effects by modeling the momentum distributions and removal energy of nucleons in nuclear targets. Functions that describe the momentum distributions and removal energy of nucleons from nuclei are referred to as spectral functions.

Spectral functions can take the simple form of a momentum distribution and a fixed removal energy (e.g. Fermi gas model [9-11]), or the more complicated form of a two dimensional (2D) distribution in both momentum and removal energy (e.g. Benhar-Fantoni spectral function $[12,13]$ ).

Figure 2 shows the nucleon momentum distributions in a ${ }^{12} \mathrm{C}$ nucleus for some of the spectral functions that are currently being used. The solid green line is the nucleon momentum distribution for the Fermi gas [9-11] model (labeled "Global Fermi" gas) which is currently implemented in all neutrino event generators (Eq. 30 of Appendix B). The solid black line is the projected momentum distribution of the Benhar-Fantoni [12,13] 2D spectral function as implemented in NuWro. The solid red line is the nucleon momentum distribution of the Local-Thomas-Fermi gas (LTF) model [8] which is implemented in NURWO and GiBUU.

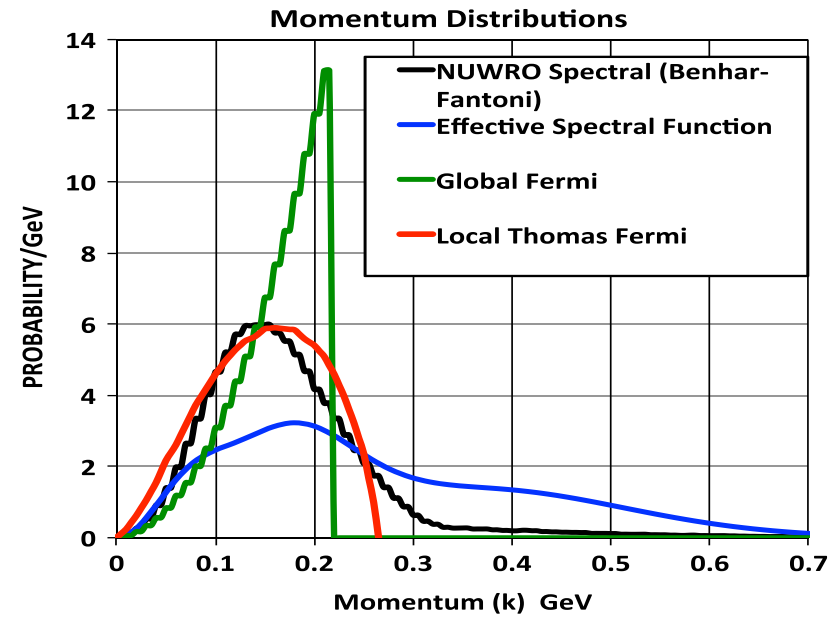

Fig. 2 Nucleon momentum distributions in a ${ }^{12} \mathrm{C}$ nucleus for several spectral functions. The curve labeled "Global Fermi" gas is the momentum distribution for the Fermi gas model (Eq. 30 in Appendix B). The blue line is the momentum distribution for the effective spectral function described in this paper

It is known that theoretical calculations using spectral functions do not fully describe the shape of the quasielastic peak for electron scattering on nuclear targets. This is because the calculations only model the initial state (shown on the top panel of Fig. 1), and do not account for final state interactions of the first kind (shown on the bottom panel of Fig. 1). Because FSI changes the amplitude of the scattering, it modifies the shape of $\frac{1}{\sigma} \frac{d \sigma}{d \nu}$. FSI reduces the cross section at the peak and increases the cross section at the tails of the distribution.

In contrast to the spectral function formalism, predictions using the $\psi^{\prime}$ superscaling formalism $[14,15]$ fully describe the longitudinal response function of quasielastic electron scattering data on nuclear targets. This is expected since the calculations use a $\psi^{\prime}$ superscaling function which is directly extracted from the longitudinal component of measured electron scattering quasielastic differential cross sections.

However, although $\psi^{\prime}$ superscaling provides a very good description of the final state lepton in QE scattering, $\psi^{\prime}$ superscaling is not implemented as an option in neutrino MC event generators that are currently used in neutrino experiments. There are specific technical issues that are associated with implementing any theoretical model within the framework of a MC generator. In addition, $\psi^{\prime}$ superscaling does not provide a detailed description of the composition of the hadronic final state. Therefore, it must also be combined with other models to include details about the composition of the hadronic final state.

Because the machinery to model both the leptonic and hadronic final state for various spectral functions is already implemented in all neutrino MC generators, adding another spectral function as an option can be implemented in a few 
days. In this communication we present the parameters for a new effective spectral function that reproduces the kinematics of the final state lepton predicted by $\psi^{\prime}$ superscaling. The momentum distribution for this ESF for ${ }^{12} \mathrm{C}$ is shown as the blue line in Fig. 2.

\subsection{The $\psi^{\prime}$ superscaling functions for QE scattering}

The $\psi$ scaling variable $[14,15]$ is defined as:

$\psi \equiv \frac{1}{\sqrt{\xi_{F}}} \frac{\lambda-\tau}{\sqrt{(1+\lambda) \tau+\kappa \sqrt{\tau(1+\tau)}}}$,

where $\xi_{F} \equiv \sqrt{1+\eta_{F}^{2}}-1, \eta_{F} \equiv K_{F} / M_{n}, \lambda \equiv v / 2 M_{n}$, $\kappa \equiv|\mathbf{q}| / 2 M_{n}$ and $\tau \equiv\left|Q^{2}\right| / 4 M_{n}^{2}=\kappa^{2}-\lambda^{2}$.

The $\psi^{\prime}$ superscaling variable includes a correction that accounts for the removal energy from the nucleus. This is achieved by replacing $v$ with $v-E_{\text {shift }}$, which forces the maximum of the $\mathrm{QE}$ response to occur at $\psi^{\prime}=0$. This is equivalent to taking $\lambda \rightarrow \lambda^{\prime}=\lambda-\lambda_{\text {shift }}$ with $\lambda_{\text {shift }}=$ $E_{\text {shift }} / 2 M_{n}$ and correspondingly $\tau \rightarrow \tau^{\prime}=\kappa^{2}-\lambda^{\prime 2}$ in Eq. (1). QE scattering on all nuclei (except for the deuteron) is described using the same universal superscaling function. The only parameters which are specific to each nucleus are the Fermi broadening parameter $K_{F}$ and the energy shift parameter $E_{\text {shift }}$.

Figure 3 shows two parametrizations of $\psi^{\prime}$ superscaling functions extracted from quasielastic electron scattering data on ${ }^{12} \mathrm{C}$. Shown is the $\psi^{\prime}$ superscaling distribution extracted from a fit to electron scattering data used by Bosted and Mamyan [15] (solid black line labeled as 2012), and the superscaling function extracted from a recent updated fit [16] to data from a large number of quasielastic electron scattering experiments on ${ }^{12} \mathrm{C}$ (dotted red line labeled as 2014). The panel on top shows the superscaling functions on a linear scale and the panel on the bottom shows the same superscaling functions on a logarithmic scale.

The $2014 \psi^{\prime}$ superscaling function is given by:

$$
F\left(\psi^{\prime}\right)=\frac{1.3429}{K_{F}\left[1+1.7119^{2}\left(\psi^{\prime}+0.19525\right)^{2}\right]\left(1+e^{-1.69 \psi^{\prime}}\right)}
$$

The $2012 \psi^{\prime}$ superscaling function is given by:

$$
F\left(\psi^{\prime}\right)=\frac{1.5576}{K_{F}\left[1+1.7720^{2}\left(\psi^{\prime}+0.3014\right)^{2}\right]\left(1+e^{-2.4291 \psi^{\prime}}\right)}
$$

For both the 2012 and 2014 parametrizations the values of the Fermi motion parameter $K_{F}$ and energy shift parameter $E_{\text {shift }}$ (given in Table 1) are taken from ref. [15].

The $\psi^{\prime}$ superscaling function is extracted from the longitudinal $\mathrm{QE}$ cross section for $Q^{2}>0.3 \mathrm{GeV}^{2}$ where there are no Pauli blocking effects. At very low values of $Q^{2}$, the $\mathrm{QE}$

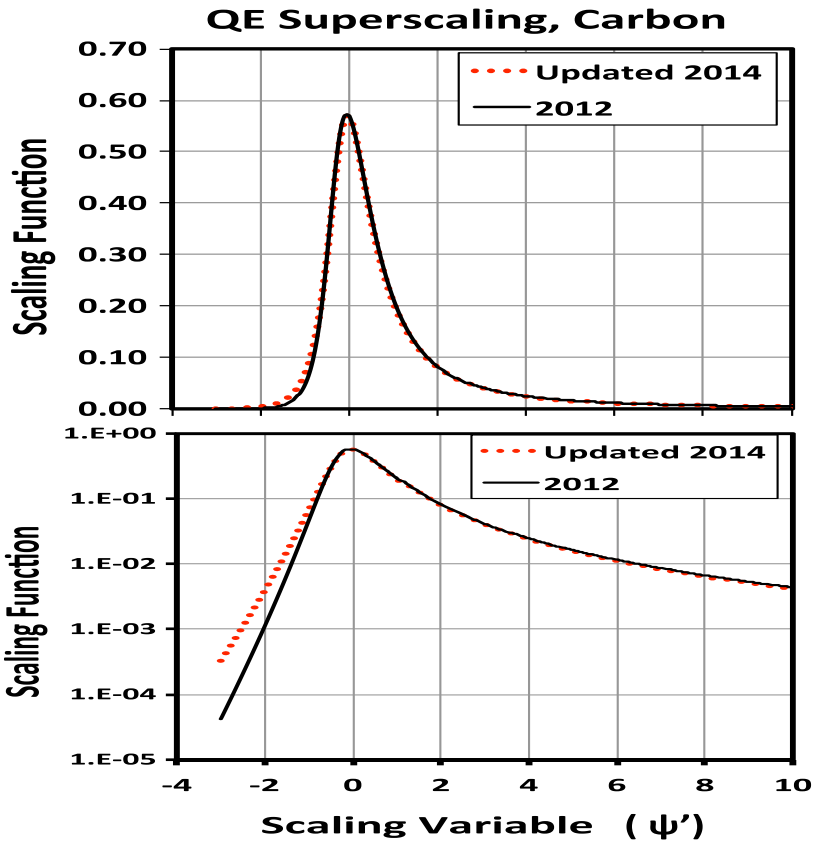

Fig. 3 The $\psi^{\prime}$ superscaling distribution extracted from a fit to electron scattering data used by Bosted and Mamyan [15] (solid black line labeled as 2012), and the superscaling function extracted from a more recent updated fit [16] to data from a large number of quasielastic electron scattering experiments on ${ }^{12} \mathrm{C}$ (dotted red line labeled as 2014). The panel on top shows the superscaling functions on a linear scale and the panel on the bottom shows the same superscaling functions on a logarithmic scale. The integral of the curve has been normalized to unity

Table 1 Values of Fermi-broadening parameter $K_{F}$ and energy shift $E_{\text {shift }}$ used in the $\psi^{\prime}$ superscaling prediction for different nuclei. The parameters for deuterium $(\mathrm{A}=2)$ are to be taken as a crude approximation only, and deuterium is treated differently as discussed in Sect. 5

\begin{tabular}{lll}
\hline$A$ & $K_{F}\left(\psi^{\prime}\right)(\mathrm{GeV})$ & $E_{\text {shift }}\left(\psi^{\prime}\right)(\mathrm{GeV})$ \\
\hline 2 & 0.100 & 0.001 \\
3 & 0.115 & 0.001 \\
$3<A<8$ & 0.190 & 0.017 \\
$7<A<17$ & 0.228 & 0.0165 \\
$16<A<26$ & 0.230 & 0.023 \\
$25<A<39$ & 0.236 & 0.018 \\
$38<A<56$ & 0.241 & 0.028 \\
$55<A<61$ & 0.241 & 0.023 \\
$A>60$ & 0.245 & 0.018 \\
\hline
\end{tabular}

differential cross sections predicted by the $\psi^{\prime}$ superscaling should be multiplied by a Pauli blocking factor $K_{\text {Pauli }}^{\text {nucle }}\left(Q^{2}\right)$ which reduces the predicted cross sections at low $Q^{2}$. The Pauli suppression factor (see Fig. 20) is given [15] by the function

$$
K_{\text {Pauli }}^{\text {nucle }}=\frac{3}{4} \frac{|\mathbf{q}|}{K_{F}}\left(1-\frac{1}{12}\left(\frac{|\mathbf{q}|}{K_{F}}\right)^{2}\right)
$$


For $|\mathbf{q}|<2 K_{F}$, otherwise no Pauli suppression correction is made. Here $|\mathbf{q}|=\sqrt{Q^{2}+v^{2}}$ is the absolute magnitude of the momentum transfer to the target nucleus,

In this paper we show that the normalized differential quasielastic cross section $\frac{1}{\sigma} \frac{d \sigma}{d \nu}\left(Q^{2}, \nu\right)$ predicted by the $\psi^{\prime}$ superscaling formalism can be well described by predictions of a modified effective spectral function (ESF). The parameters of the ESF are obtained by requiring that the ESF predictions for $\frac{1}{\sigma} \frac{d \sigma}{d v}\left(Q^{2}, v\right)$ at $Q^{2}$ values of $0.1,0.3,0.5$ and 0.7 $\mathrm{GeV}^{2}$ be in agreement with the predictions of the $2014 \psi^{\prime}$ superscaling function given in Eq. 3 .

The predictions of the $\psi^{\prime}$ formalism are given by

$\frac{1}{\sigma} \frac{d \sigma}{d \nu}\left(Q^{2}, \nu\right)=\frac{1}{N} F\left(\psi^{\prime}\right)$

where $\mathrm{N}$ the integral of $F\left(\psi^{\prime}\right) d v$ over all values of $v$ (for a given value of $Q^{2}$ ).

\subsection{Comparison of models for quasielastic scattering}

For electron scattering, the nuclear response function is extracted from the normalized longitudinal differential cross section at a fixed vale of $Q^{2}\left(\frac{1}{\sigma} \frac{d \sigma}{d v}\left(Q^{2}, v\right)\right)$. Here $\sigma$ is the integral of $\frac{d \sigma}{d \nu}$ for a fixed value of $Q^{2}$. The normalization removes the effects of the $Q^{2}$-dependent nucleon vector form factors. In models which assume scattering from independent nucleons, the response functions for the longitudinal and transverse $\mathrm{QE}$ cross sections are the same.

For neutrino scattering at high energy, the QE cross section is dominated by the structure function $W_{2}$. Therefore, in models which assume scattering from independent nucleons the normalized cross section $\left(\frac{1}{\sigma} \frac{d \sigma}{d v}\left(Q^{2}, v\right)\right)$ at a fixed value of $Q^{2}$ at high neutrino energy is also equal to the nuclear response function.

For the neutrino case, the normalization removes the effects of the $Q^{2}$-dependent nucleon vector and axial form factors. Figure 4 shows predictions for the normalized QE differential cross sections $\frac{1}{\sigma} \frac{d \sigma}{d v}\left(Q^{2}, \nu\right)$ for $10 \mathrm{GeV}$ neutrinos on ${ }^{12} \mathrm{C}$ at $Q^{2}=0.5 \mathrm{GeV}^{2}$ for various spectral functions. Here $\frac{1}{\sigma} \frac{d \sigma}{d v}$ is plotted versus $\Delta v=v-\frac{Q^{2}}{2 M_{p}}$. The prediction of the $\psi^{\prime}$ superscaling formalism for $\frac{1}{\sigma} \frac{d \sigma}{d \nu}\left(Q^{2}, \nu\right)$ is shown as the solid black line. The solid green line is the prediction using the "Global Fermi" gas [9-11] momentum distribution given in Appendix B (eq. 33). The solid red line is the prediction using the Local Thomas Fermi gas (LTF) momentum distribution. The dotted purple line is the NuWro prediction using the full two dimensional Benhar-Fantoni $[12,13]$ spectral function. The predictions of all of these spectral functions for $\frac{1}{\sigma} \frac{d \sigma}{d v}\left(Q^{2}, \nu\right)$ are in disagreement with the predictions of the $\psi^{\prime}$ superscaling formalism.

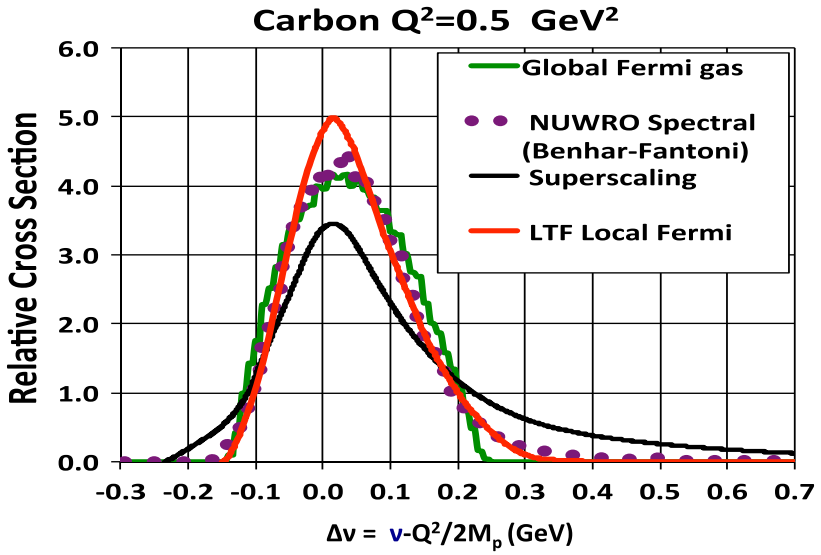

Fig. 4 Comparison of the $\psi^{\prime}$ superscaling prediction (solid black line) for the normalized $\frac{1}{\sigma} \frac{d \sigma}{d v}\left(Q^{2}, v\right)$ at $Q^{2}=0.5 \mathrm{GeV}^{2}$ for $10 \mathrm{GeV}$ neutrinos on ${ }^{12} \mathrm{C}$ to the predictions of several spectral function models. Here $\frac{1}{\sigma} \frac{d \sigma}{d v}\left(Q^{2}, v\right)$ is plotted versus $\Delta v$ The curve labeled "Global Fermi" gas is the distribution for the Fermi gas model given in Appendix B (Eq. 33). The predictions of the spectral function models are in disagreement with the predictions of $\psi^{\prime}$ superscaling

\section{Effective spectral function for ${ }^{12} \mathrm{C}$}

\subsection{Momentum distribution}

The probability distribution for a nucleon to have a momentum $k=|\mathbf{k}|$ in the nucleus is defined as

$P(k) d k=4 \pi k^{2}|\phi(k)|^{2} d k$.

For $k<0.65 \mathrm{GeV}$, we parametrize [19] $P(k)$ by the following function:

$P(k)=\frac{\pi}{4 c_{0}} \frac{1}{N}\left(a_{s}+a_{p}+a_{t}\right) y^{2}$

where

$$
\begin{aligned}
y & =\frac{k}{c_{0}} \\
a_{s} & =c_{1} e^{-\left(b_{s} y\right)^{2}} \\
a_{p} & =c_{2}\left(b_{p} y\right)^{2} e^{-\left(b_{p} y\right)^{2}} \\
a_{t} & =c_{3} y^{\beta} e^{-\alpha(y-2)}
\end{aligned}
$$

For $k>0.65 \mathrm{GeV}$ we set $P(k)=0$. Here, $c_{0}=0.197, k$ is in $\mathrm{GeV}, \mathrm{N}$ is a normalization factor to normalize the integral of the momentum distribution from $k=0$ to $k=0.65 \mathrm{GeV}$ to 1.0 , and $\mathrm{P}(k)$ is in units of $\mathrm{GeV}^{-1}$. The parameters that describe the projected momentum distribution [19] for the BenharFantoni $[12,13]$ spectral function for nucleons bound in ${ }^{12} \mathrm{C}$ are given in the second column of Table 2 .

\subsection{Removal energy}

The kinematics for neutrino charged current quasielastic scattering from a off-shell bound neutron with momentum $\mathbf{k}$ and 
Table 2 A comparison of the parameters that describe the projected momentum distribution for the Benhar-Fantoni spectral function for nucleons bound in ${ }^{12} \mathrm{C}$ ( 2 nd column) with the parameters that describe the effective spectral function (ESF) for ${ }^{12} \mathrm{C}$ ( $3 \mathrm{rd}$ column). Here, $\Delta$ is the average binding energy parameter of the spectator one-hole nucleus for the $1 \mathrm{p} 1 \mathrm{~h}$ process and $f_{1 p 1 h}$ is the fraction of the scattering that occurs via the $1 \mathrm{p} 1 \mathrm{~h}$ process. For the $2 \mathrm{p} 2 \mathrm{~h}$ process the average binding energy for the two-hole spectator nucleus is $2 \Delta$. The parameters for the effective spectral function for deuterium $\left({ }^{2} \mathrm{H}\right)$ are given in the 4th column

\begin{tabular}{llll}
\hline Parameter & Benhar-Fantoni & ESF & ESF \\
\hline Nucleus & ${ }^{12} \mathrm{C}$ & ${ }^{12} \mathrm{C}$ & ${ }^{2} \mathrm{H}$ \\
$\Delta(\mathrm{MeV})$ & 2 Dspectral & 12.5 & 0.13 \\
$f_{1 p 1 h}$ & 2 Dspectral & 0.808 & 0 \\
$f_{2 p 2 h}$ & 2 Dspectral & 0.192 & 1.00 \\
$b_{s}$ & 1.7 & 2.12 & 0.413475 \\
$b_{p}$ & 1.77 & 0.7366 & 1.75629 \\
$\alpha$ & 1.5 & 12.94 & 8.29029 \\
$\beta$ & 0.8 & 10.62 & $3.621 \times 10^{-3}$ \\
$c_{1}$ & 2.823397 & 197.0 & 0.186987 \\
$c_{2}$ & 7.225905 & 9.94 & 6.24155 \\
$c_{3}$ & 0.00861524 & $4.36 \times 10^{-5}$ & $2.082 \times 10^{-4}$ \\
$N$ & 0.985 & 29.64 & 10.33 \\
\hline
\end{tabular}

energy $E_{n}$ are given by:

$$
\begin{aligned}
\left(M_{n}^{\prime}\right)^{2} & =\left(E_{n}\right)^{2}-V k^{2} \\
M_{p}^{2} & =\left(M_{n}^{\prime}\right)^{2}+2 E_{n} v-2|\mathbf{q}| k_{z}-Q^{2} \\
v & =E_{v}-E_{\mu}=\frac{Q^{2}+M_{p}^{2}-\left(M_{n}^{\prime}\right)^{2}+2|\mathbf{q}| k_{z}}{\left(E_{n}\right)} \\
V\left(Q^{2}\right) & =1-e^{-x Q^{2}}, x=12.04
\end{aligned}
$$

For scattering from a single off-shell nucleon, the term $V\left(Q^{2}\right)$ multiplying $k^{2}$ in Eqs. 6, 8 and 9 (and also Eqs. 21, 22, and 23) should be 1.0. However, we find that in order to make the spectral function predictions agree with $\psi^{\prime}$ superscaling at very low $Q^{2}$ (e.g. $Q^{2}<0.3 \mathrm{GeV}^{2}$ ) we need to apply a $Q^{2}$-dependent correction to reduce the removal energy, e.g. due to final state interaction (of the first kind) at low $Q^{2}$. This factor is given in Eq. 7 and plotted in Fig. 5.

The value of the parameter $x=12.04 \mathrm{GeV}^{-2}$ was extracted from the fits discussed in section 2.2.4. As mentioned earlier, $\mathbf{q}$ is the momentum transfer to the neutron. We define the component of the initial neutron momentum $\mathbf{k}$ which is parallel to $\mathbf{q}$ as $k_{z}$. The expression for $E_{n}$ depends on the process and is given by Eqs. 8 and 9 for the $1 \mathrm{p} 1 \mathrm{~h}$, and $2 \mathrm{p} 2 \mathrm{~h}$ process, respectively.

We assume that the off-shell energy $\left(E_{n}\right)$ for a bound neutron with momentum $\mathbf{k}$ can only take two possible values

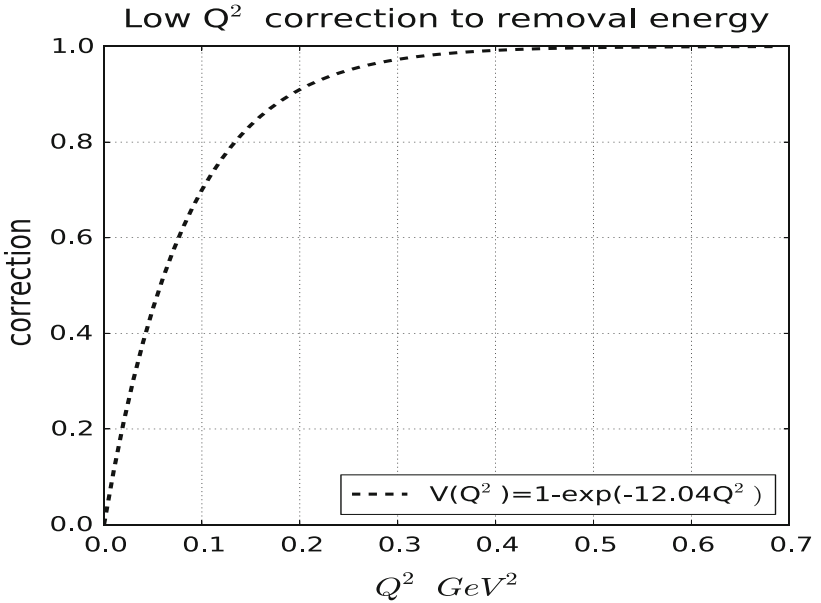

Fig. 5 The $Q^{2}$-dependent correction that accounts for the reduction of the removal energy at low $Q^{2}$, e.g. due to final state interaction (of the first kind)

$[17,18]$. We refer to the first possibility as the $1 \mathrm{p} 1 \mathrm{~h}$ process (one proton, one hole in the final state). The second possibility is the $2 \mathrm{p} 2 \mathrm{~h}$ process (two protons and two holes in the final state).

In our effective spectral function model the $1 \mathrm{p} 1 \mathrm{~h}$ process occurs with probability $f_{1 p 1 h}$, and the $2 \mathrm{p} 2 \mathrm{~h}$ process occurs with probability of $1-f_{1 p 1 h}$. For simplicity, we assume that the probability $f_{1 p 1 h}$ is independent of the momentum of the bound nucleon.

\subsubsection{The 1plh process}

The $1 \mathrm{p} 1 \mathrm{~h}$ process refers to scattering from an independent neutron in the nucleus resulting in a final state proton and a hole in the spectator nucleus. Figure 1 illustrates the $1 \mathrm{p} 1 \mathrm{~h}$ process (for $Q^{2}>0.3 \mathrm{GeV}^{2}$ ), for the scattering from an offshell bound neutron of momentum $-\mathbf{k}$ in a nucleus of mass A $[17,18]$. In the $1 \mathrm{p} 1 \mathrm{~h}$ process, momentum is balanced by an on-shell recoil $[A-1]^{*}$ nucleus which has momentum $\mathbf{P}_{\mathbf{A}-\mathbf{1}}^{*}=\mathbf{P}_{\mathbf{S}}=\mathbf{k}$ and an average binding energy parameter $\Delta$, where $M_{A}-M_{A-1}^{*}=M_{n}+\Delta$. The initial state off-shell neutron has energy $E_{n}$ which is given by:

$$
\begin{aligned}
E_{n}(1 p 1 h) & =M_{A}-\sqrt{V k^{2}+\left(M_{A-1}^{*}\right)^{2}} \\
& \approx M_{n}-\Delta-\frac{V k^{2}}{2 M_{A-1}^{*}}
\end{aligned}
$$

The final state includes a proton and an $[A-1]^{*}$ nucleus which is in an excited state because the removal of the nucleon leaves hole in the energy levels of the nucleus.

For the $1 \mathrm{p} 1 \mathrm{~h}$ process, the removal energy of a nucleon includes the following two contributions: 


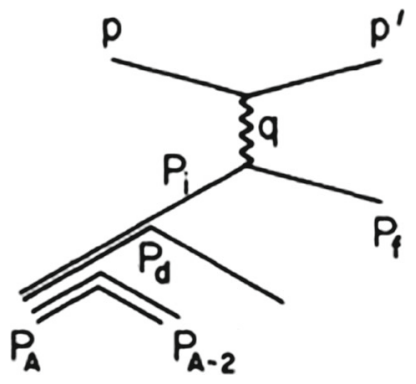

Fig. 6 2p2h process: Scattering from an off-shell bound neutron of momentum $\mathbf{P}_{\mathbf{i}}=-\mathbf{k}$ from two nucleon correlations (quasi-deuteron). The on-shell recoil spectator nucleon has momentum $\mathbf{P}_{\mathbf{s}}=\mathbf{k}$

- The binding energy parameter $\Delta$ where $M_{A}-M_{A-1}^{*}=$ $M_{n}+\Delta$.

- The kinetic energy of the recoil spectator nucleus $\frac{V k^{2}}{2 M_{A-1}^{*}}$.

\subsubsection{The $2 p 2 h$ process}

In general, there are are several processes which result in two (or more) nucleons and a spectator excited nucleus with two (or more) holes in final state:

- Two nucleon correlations in initial state (quasi deuteron) which are often referred to as short range correlations (SRC).

- Final state interaction (of the first kind) resulting in a larger energy transfer to the hadronic final state (as modeled by superscaling).

- Enhancement of the transverse cross sections ("Transverse Enhancement") from meson exchange currents (MEC) and isobar excitation.

In the effective spectral function approach the lepton energy spectrum for all three processes is modeled as originating from the two nucleon correlation process. This accounts for the additional energy shift resulting from the removal of two nucleons from the nucleus.

Figure 6 illustrates the $2 \mathrm{p} 2 \mathrm{~h}$ process for scattering from an off-shell bound neutron of momentum $-\mathbf{k}$ (for $Q^{2}>0.3$ $\mathrm{GeV}^{2}$ ). The momentum of the interacting nucleon in the initial state is balanced by a single on-shell correlated recoil nucleon which has momentum $\mathbf{k}$. The $[A-2]^{*}$ spectator nucleus is left with two holes. The initial state off-shell neutron has energy $E_{n}$ which is given by:

$E_{n}(2 p 2 h)=\left(M_{p}+M_{n}\right)-2 \Delta-\sqrt{V k^{2}+M_{p}^{2}}$

where $V$ is given by Eq. 7 .

For the $2 \mathrm{p} 2 \mathrm{~h}$ process, the removal energy of a nucleon includes the following two contributions:

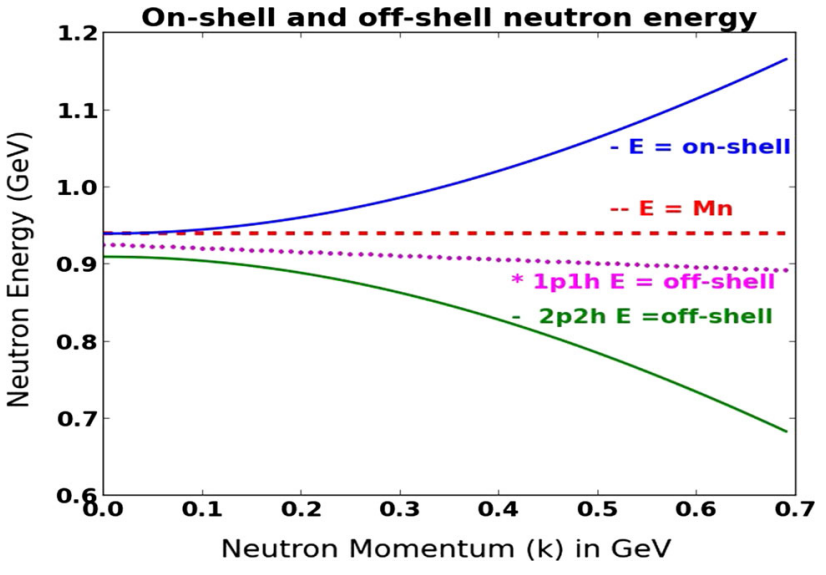

Fig. 7 Comparison of energy for on-shell and off-shell bound neutrons in ${ }^{12} \mathrm{C}$. The on-shell energy is $E_{n}=\sqrt{k^{2}+M_{n}^{2}}$. The off-shell energy is shown for both the $1 \mathrm{p} 1 \mathrm{~h}\left(E_{n}=M_{n}-\Delta-\frac{V k^{2}}{2 M_{A-1}^{*}}\right)$ and $2 \mathrm{p} 2 \mathrm{~h}$ process $\left(E_{n}=\left(M_{p}+M_{n}\right)-2 \Delta-\sqrt{V k^{2}+M_{p}^{2}}\right.$, where $\left(M_{p}+M_{n}\right)$ and $\Delta$ is the average binding energy parameter of the spectator one-hole nucleus. Shown is the case with $\mathrm{V}=1$. (The factor $\mathrm{V}$ is given in Eq. 7 and plotted in Fig. 5. $\mathrm{V} \approx 1$ for $Q^{2}>0.3 \mathrm{GeV}^{2}$ )

- The binding energy parameter $2 \Delta$ where $M_{A}-M_{A-2}^{*}=$ $M_{n}+M_{p}+2 \Delta$.

- The kinetic energy of the recoil spectator nucleon given by $\sqrt{V k^{2}+M_{p}^{2}}$.

Figure 7 shows a comparison of the total energy for onshell and off-shell bound neutrons in ${ }^{12} \mathrm{C}$ as a function of neutron momentum $\mathrm{k}$ (for $Q^{2}>0.3 \mathrm{GeV}^{2}$ where $\mathrm{V} \approx 1.0$ ). The energy for an unbound on-shell neutron is $E_{n}=\sqrt{V k^{2}+M_{n}^{2}}$. The off-shell energy of a bound neutron is shown for both the $1 \mathrm{p} 1 \mathrm{~h}\left(E_{n}=M_{n}-\Delta-\frac{\mathbf{V k}^{\mathbf{2}}}{2 M_{A-1}^{*}}\right)$ and the $2 \mathrm{p} 2 \mathrm{~h}$ process $\left(E_{n}=\left(M_{p}+M_{n}\right)-2 \Delta-\sqrt{V k^{2}+M_{p}^{2}}\right)$.

In the effective spectral function approach, all effects of final state interaction (of the first kind) are absorbed in the initial state effective spectral function. The parameters of the effective spectral function are obtained by finding the parameters $x, \Delta, f_{1 p 1 h}, b_{s}, b_{p}, \alpha, \beta, c_{1}, c_{2}, c_{3}$ and $N$ for which the predictions of the effective spectral function best describe the predictions of the $\psi^{\prime}$ superscaling formalism for $(1 / \sigma) d \sigma / d v$ at $Q^{2}$ values of $0.1,0.3,0.5$ and $0.7 \mathrm{GeV}^{2}$.

Figure 8 compares predictions for $\frac{1}{\sigma} \frac{d \sigma}{d v}\left(Q^{2}, v\right)$ for ${ }^{12} \mathrm{C}$ as a function of $\Delta v$ at $Q^{2}=0.5 \mathrm{GeV}^{2}$. The prediction of the effective spectral function is the dashed blue curve. The prediction of the $\psi^{\prime}$ superscaling model is the solid black curve. For $Q^{2}=0.5 \mathrm{GeV}^{2}$ the prediction of the effective spectral function is almost identical to the prediction of $\psi^{\prime}$ superscaling. All of the prediction for the effective spectral function are calculated from Eq. 28 in Appendix B. 


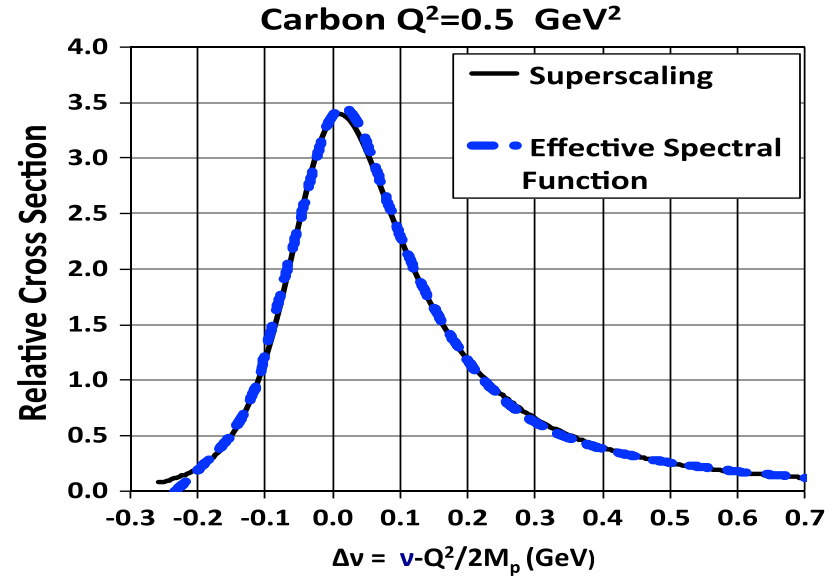

Fig. 8 Comparison of the prediction for the normalized QE differential cross section $\left(\frac{1}{\sigma} \frac{d \sigma}{d v}\left(Q^{2}, v\right)\right)$ for ${ }^{12} \mathrm{C}$ from the effective spectral function to the prediction of $\psi^{\prime}$ superscaling. The predictions are shown as a function of $\Delta v$ at $Q^{2}=0.5 \mathrm{GeV}^{2}$. The prediction of the effective spectral function are calculated from Eq. 28 in Appendix B. For $Q^{2}=$ $0.5 \mathrm{GeV}^{2}$ the prediction of the effective spectral function are almost identical to the prediction of $\psi^{\prime}$ superscaling

For the $2 \mathrm{p} 2 \mathrm{~h}$ process, each of the two final state nucleons can also undergo final state interactions (of the second kind) with other nucleons in the spectator $[A-2]^{*}$ nucleus.

\subsubsection{Comparison of the $1 p 1 h$ and $2 p 2 h$ contributions}

The top panel of Fig. 9 shows the prediction of the effective spectral function model for $\frac{1}{\sigma} \frac{d \sigma}{d v}\left(Q^{2}, v\right)$ for QE scattering from a ${ }^{12} \mathrm{C}$ nucleus at $Q^{2}=0.5 \mathrm{GeV}^{2}$, assuming that only the $1 \mathrm{p} 1 \mathrm{~h}$ process contributes. The bottom panel of Fig. 9 shows the prediction of the effective spectral function model for $\frac{1}{\sigma} \frac{d \sigma}{d v}\left(Q^{2}, v\right)$ for $\mathrm{QE}$ scattering from a ${ }^{12} \mathrm{C}$ nucleus at $Q^{2}$ $=0.5 \mathrm{GeV}^{2}$ assuming that only the $2 \mathrm{p} 2 \mathrm{~h}$ process contributes.

We find that the effective spectral function with only the $1 \mathrm{p} 1 \mathrm{~h}$ process provides a reasonable description of the prediction of $\psi^{\prime}$ superscaling. Including a contribution from the $2 \mathrm{p} 2 \mathrm{~h}$ process in the fit improves the agreement and results in a prediction which is almost identical to the prediction of $\psi^{\prime}$ superscaling.

For reference, figures 9-12 also show the prediction for the Fermi Gas model in blue, and the predictions from the Bodek-Ritchie $[17,18]$ Fermi gas model which includes a high momentum contribution from two nucleon correlations in green. These predictions are calculated for $10 \mathrm{GeV}$ neutrinos using the the GENIE neutrino Monte Carlo generator.

\subsubsection{Comparisons as a function of $Q^{2}$ for $Q^{2}>0.3 \mathrm{GeV}^{2}$}

Figures 10 and 11 show a comparison of the prediction of the effective spectral function for $\frac{1}{\sigma} \frac{d \sigma}{d v}\left(Q^{2}, v\right)$ for ${ }^{12} \mathrm{C}$ to the
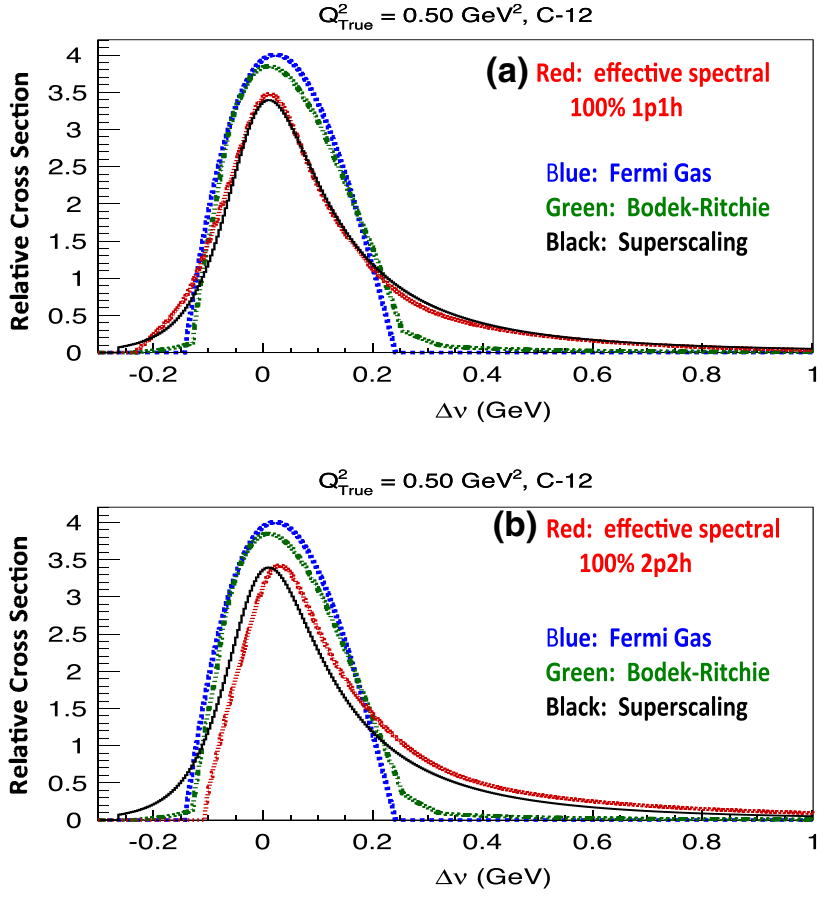

Fig. 9 Comparison of the two contributions to the normalized QE cross section $\left(\frac{1}{\sigma} \frac{d \sigma}{d v}\left(Q^{2}, v\right)\right)$ for ${ }^{12} \mathrm{C}$ from the effective spectral function. (a) For the $1 \mathrm{p} 1 \mathrm{~h}$ component, (b) For the $2 \mathrm{p} 2 \mathrm{~h}$ component. Here, each contribution (shown in red) is normalized to 1.0. Also shown (for reference) are the predictions for superscaling (black line), for the Fermi Gas model (blue), and the predictions from the Bodek-Ritchie [17,18] Fermi gas model which includes a high momentum contribution from two nucleon correlations (green)

predictions of the $\psi^{\prime}$ superscaling formalism for $Q^{2}$ values of $0.3,0.5,0.7,1.0,1.2,1.5$ and 2.0 and $\mathrm{GeV}^{2}$.

In principle, it should not be possible for a spectral function approach to exactly reproduce $\psi^{\prime}$ superscaling at all values of $Q^{2}$. Nonetheless, the parameters which we optimized for $Q^{2}$ values of $0.3,0.5$ and $0.7 \mathrm{GeV}^{2}$ also provide a good description of $\frac{1}{\sigma} \frac{d \sigma}{d \nu}(\nu)$ for $Q^{2}$ values of 1.0, 1.2, 1.5 and 2.0 $\mathrm{GeV}^{2}$.

\subsubsection{Comparisons as a function of $Q^{2}$ for $Q^{2}<0.3 \mathrm{GeV}^{2}$}

The low $Q^{2}$ suppression factor of the removal energy which is given in Eq. 7 is introduced in order to reproduce predictions of the $\psi^{\prime}$ superscaling model at $Q^{2}<0.3 \mathrm{GeV}^{2}$.

Figure 12 shows a comparison of the prediction for the shape $\left(\frac{1}{\sigma} \frac{d \sigma}{d v}\left(Q^{2}, v\right)\right)$ of the quasielastic peak for ${ }^{12} \mathrm{C}$ from the effective spectral function (red) to the predictions of the $\psi^{\prime}$ superscaling model (black). The predictions are shown as a function of $\Delta v$ for $Q^{2}=0.1 \mathrm{GeV}^{2}$. The top panel show the predictions without the low $Q^{2}$ correction factor to the removal energy. The bottom panel shows the predictions including the low $Q^{2}$ correction factor to the removal energy, e.g. from final state interaction (of the first kind) at low $Q^{2}$. 

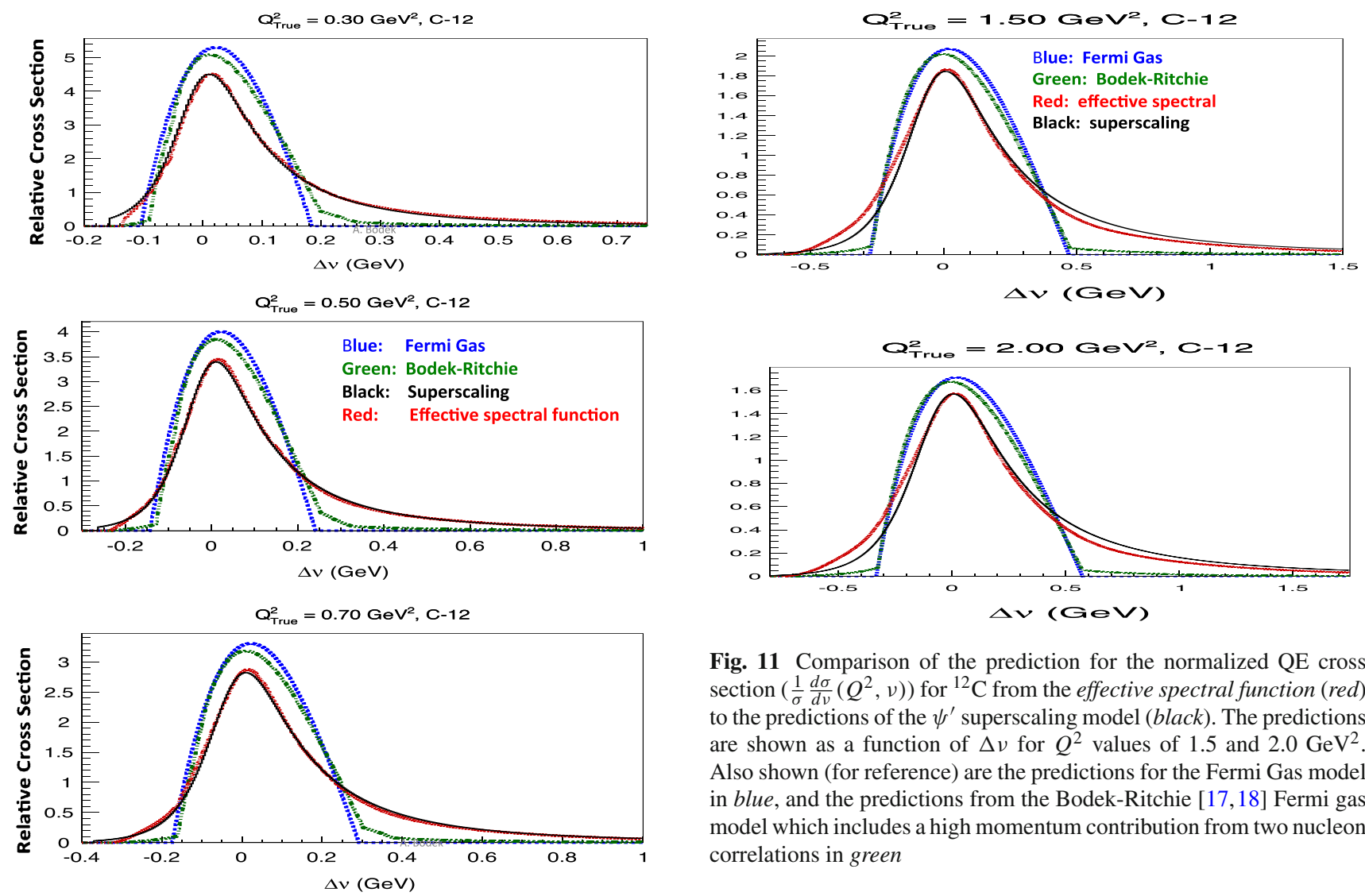

Fig. 11 Comparison of the prediction for the normalized QE cross section $\left(\frac{1}{\sigma} \frac{d \sigma}{d v}\left(Q^{2}, \nu\right)\right)$ for ${ }^{12} \mathrm{C}$ from the effective spectral function (red) to the predictions of the $\psi^{\prime}$ superscaling model (black). The predictions are shown as a function of $\Delta v$ for $Q^{2}$ values of 1.5 and $2.0 \mathrm{GeV}^{2}$. Also shown (for reference) are the predictions for the Fermi Gas model in blue, and the predictions from the Bodek-Ritchie [17,18] Fermi gas model which includes a high momentum contribution from two nucleon correlations in green

Also shown (for reference) is the prediction for superscaling in black, the prediction for the Fermi Gas model in blue, and the predictions from the Bodek-Ritchie $[17,18]$ Fermi gas model which includes a high momentum contribution from two nucleon correlations in green.

\section{Spectral functions for other nuclei}

Figure 13 shows the predictions for the shape of the quasielastic peak $\left(\frac{1}{\sigma} \frac{d \sigma}{d v}(v)\right)$ from the best fit effective spectral function (top panel) as compared to the predictions of $\psi^{\prime}$ superscaling model (bottom panel) for different nuclei. The predictions are shown as a function of $\Delta v$ for $Q^{2}=0.5 \mathrm{GeV}^{2}$. Figure 14 shows the momentum distribution of the effective spectral function for various nuclei, and Table 3 gives the parameterizations of the effective spectral function for various nuclei.

Fig. 10 Comparison of the prediction for the normalized QE cross section $\left(\frac{1}{\sigma} \frac{d \sigma}{d v}\left(Q^{2}, v\right)\right)$ for ${ }^{12} \mathrm{C}$ from the effective spectral function (red) to the predictions of the $\psi^{\prime}$ superscaling model (black). The predictions are shown as a function of $\Delta v$ for $Q^{2}$ values of $0.3,0.5,0.7,1.0$, and $1.2 \mathrm{GeV}^{2}$. Also shown (for reference) are the predictions for the Fermi Gas model in blue, and the predictions from the Bodek-Ritchie [17,18] Fermi gas model which includes a high momentum contribution from two nucleon correlations in green

\section{Transverse enhancement and multi nucleon processes}

Both spectral functions and the $\psi^{\prime}$ superscaling formalism model QE scattering in terms of scattering from independent nucleons in the initial state. The independent nucleon approach works well in modeling the longitudinal (electric) cross section for QE electron scattering from nuclear tar- 

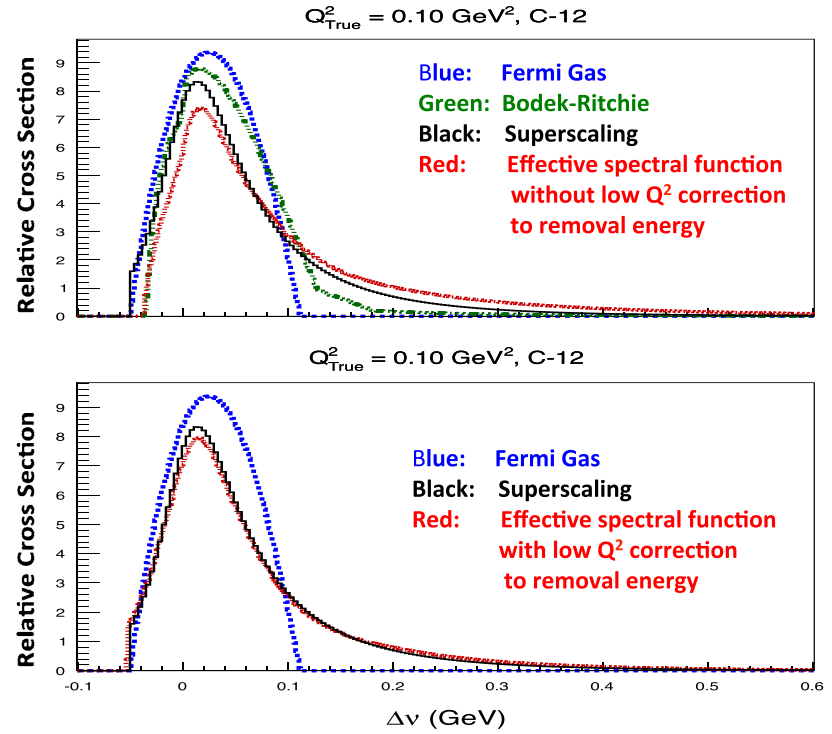

Fig. 12 Comparison of the prediction for the normalized QE cross section $\left(\frac{1}{\sigma} \frac{d \sigma}{d v}\left(Q^{2}, \nu\right)\right)$ for ${ }^{12} \mathrm{C}$ from the effective spectral function (red) to the predictions of the $\psi^{\prime}$ superscaling model (black). The predictions are shown as a function of $\Delta v$ for $Q^{2}=0.1 \mathrm{GeV}^{2}$. The top panel show the predictions without the low $Q^{2}$ correction factor to the removal energy, The bottom panel shows the predictions including the low $Q^{2}$ correction factor to the removal energy e.g. from final state interaction (of the first kind) at low $Q^{2}$. Also shown (for reference) is the prediction for superscaling in black, the predictions for the Fermi Gas model in blue, and the predictions from the Bodek-Ritchie [17,18] Fermi gas model which includes a high momentum contribution from two nucleon correlations in green
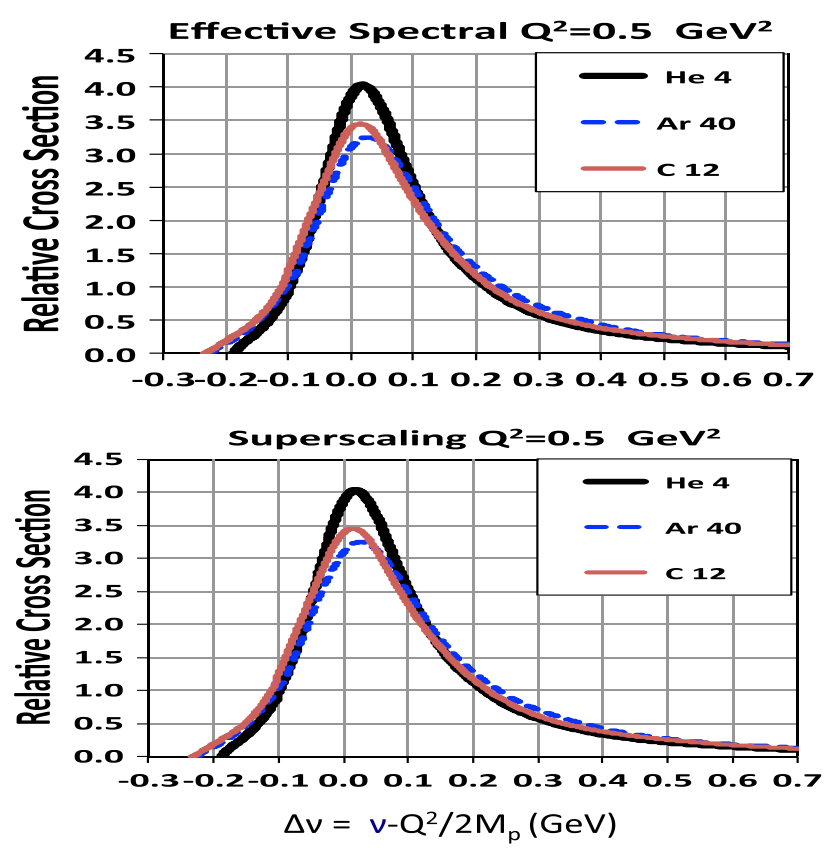

Fig. 13 Predictions for the normalized QE cross section $\left(\frac{1}{\sigma} \frac{d \sigma}{d v}\left(Q^{2}, v\right)\right)$ from the effective spectral function (top panel) as compared to the predictions of $\psi^{\prime}$ superscaling (bottom panel) for different nuclei. The predictions are shown as a function of $\Delta v$ for $Q^{2}=0.5 \mathrm{GeV}^{2}$

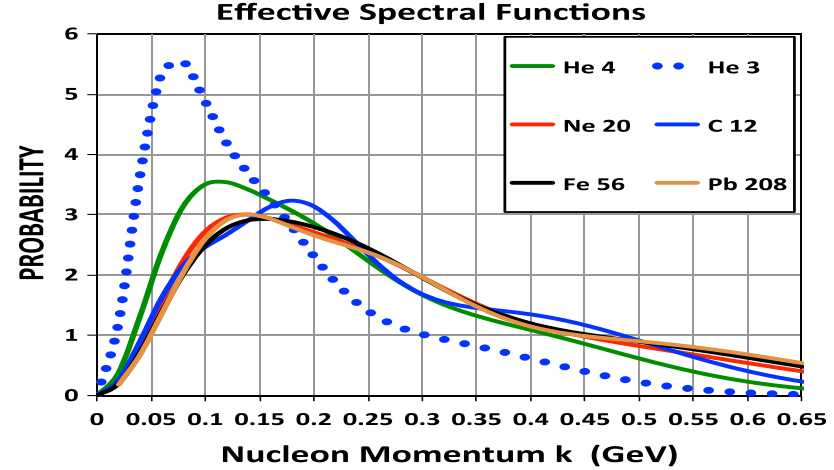

Fig. 14 The momentum distributions for the effective spectral function for various nuclei $\left({ }^{3} \mathrm{He},{ }^{4} \mathrm{He},{ }^{12} \mathrm{C}{ }^{20} \mathrm{Ne},{ }^{56} \mathrm{Fe}\right.$, and $\left.{ }^{208} \mathrm{~Pb}\right)$. In the analysis we set all distributions to zero for $k>0.65 \mathrm{GeV}$

gets. However, it is known that none of the independent nucleon models can describe the transverse (magnetic) part of the QE electron scattering cross section. The transverse cross section is larger than the predictions of the independent nucleon model, and the enhancement is a function of $Q^{2}$. This experimental observation is usually referred to as "Transverse Enhancement" (TE).

TE has been attributed to multi nucleon processes such as meson exchange currents and isobar excitation. There are two ways to account for these multi nucleon effects.

\subsection{Accounting for contributions from multi nucleon processes}

One way to account for TE is to use specific theoretical models to estimate the contributions from various multi nucleon processes such as meson exchange currents and isobar excitation. The differences between various model may provide an indication of the uncertainties of the calculations.

\subsection{The transverse enhancement (TE) model}

Another way to account for transverse enhancement is to parameterize the experimentally observed excess in the electron scattering data in a model independent way. In the TE model [27] this is done by modifying the magnetic form factors for bound nucleons.

As was done in reference [27], we have re-extracted the integrated transverse enhancement ratio $\mathcal{R}_{T}$, where

$$
\mathcal{R}_{T}=\frac{\left(Q E_{\text {transverse }}+T E\right)_{\text {exp }}}{\left(Q E_{\text {transverse }}\right)_{\text {model }}},
$$

from electron scattering data from the JUPITER collaboration [29]. Here $\left(Q E_{\text {transverse }}+T E\right)_{\text {exp }}$ is the experimentally observed integrated transverse $\mathrm{QE}$ cross section and ( $\left.Q E_{\text {transverse }}\right)_{\text {model }}$ is the integrated transverse $\mathrm{QE}$ cross section predicted by $\psi^{\prime}$ super scaling. 
Table 3 Parameterizations of the effective spectral function for various nuclei. Here, $\Delta$ is the binding energy parameter, and $f_{1 p 1 h}$ is the fraction of the scattering that occurs via the $1 p 1 h$ process. For deuterium $\left({ }^{2} \mathrm{H}\right)$ are see Table 2

\begin{tabular}{lllllllll}
\hline Parameter & ${ }^{3} \mathrm{He}$ & ${ }^{4} \mathrm{He}$ & ${ }^{12} \mathrm{C}$ & ${ }^{20} \mathrm{Ne}$ & ${ }^{27} \mathrm{Al}$ & ${ }^{40} \mathrm{Ar}$ & ${ }^{56} \mathrm{Fe}$ & 18.8 \\
\hline$\Delta(\mathrm{MeV})$ & 5.3 & 14.0 & 12.5 & 16.6 & 12.5 & 20.6 & 15.1 & 0.822 \\
$f_{1 p 1 h}$ & 0.312 & 0.791 & 0.808 & 0.765 & 0.774 & 0.809 & 1.896 \\
$b_{s}$ & 3.06 & 2.14 & 2.12 & 1.82 & 1.73 & 1.67 & 1.79 & 0.597 \\
$b_{p}$ & 0.902 & 0.775 & 0.7366 & 0.610 & 0.621 & 0.615 & 0.52 \\
$\alpha$ & 10.93 & 9.73 & 12.94 & 6.81 & 7.20 & 8.54 & 7.10 & 11.24 \\
$\beta$ & 6.03 & 7.57 & 10.62 & 6.08 & 6.73 & 8.62 & 6.26 \\
$c_{1}$ & 199.6 & 183.4 & 197.0 & 25.9 & 21.0 & 200.0 & 18.37 \\
$c_{2}$ & 1.92 & 5.53 & 9.94 & 0.59 & 0.59 & 6.25 & 0.505 \\
$c_{3}$ & $5.26 \times 10^{-5}$ & $59.0 \times 10^{-5}$ & $4.36 \times 10^{-5}$ & $221 . \times 10^{-5}$ & $121.5 \times 10^{-5}$ & $269.0 \times 10^{-5}$ & $141.0 \times 10^{-5}$ & $9.28 \times 10^{-5}$ \\
$N$ & 6.1 & 18.94 & 29.64 & 4.507 & 4.065 & 40.1 & 3.645 \\
\hline
\end{tabular}
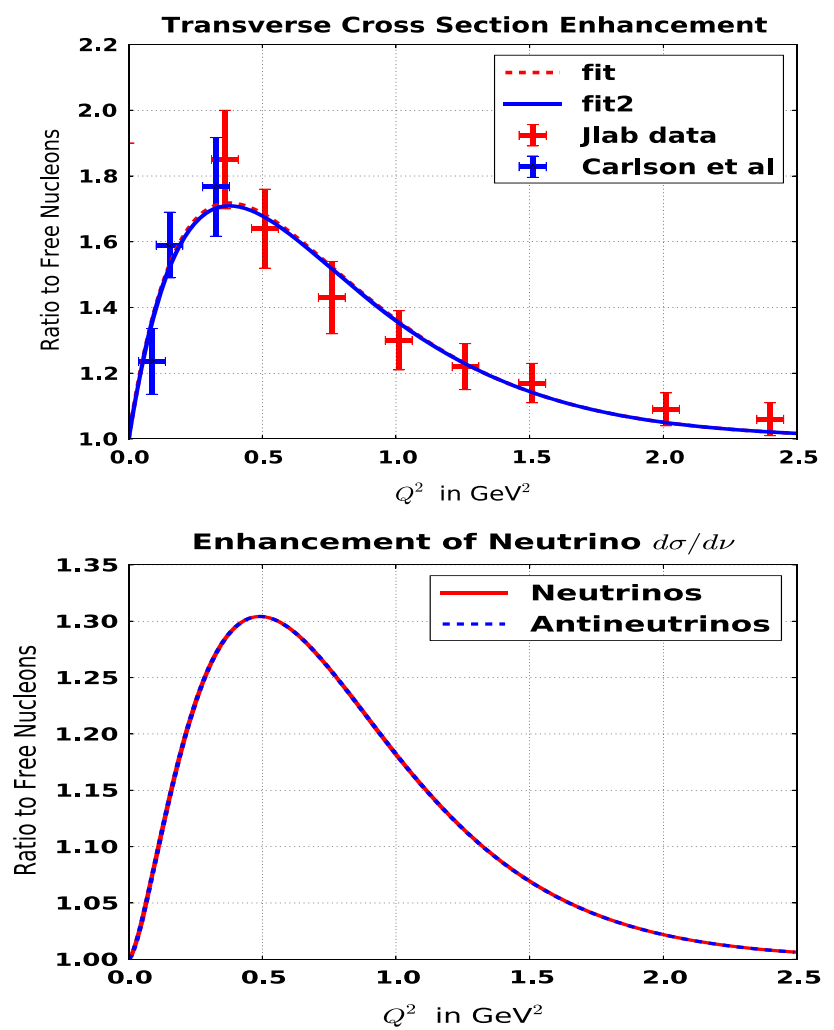

Fig. 15 Top panel: Electron scattering data. The ratio of the transverse $\mathrm{QE}$ cross section with TE to the transverse cross section for free nucleons as a function of $Q^{2}$. Bottom panel: The ratio of $d \sigma / d Q^{2}$ neutrino $\mathrm{QE}$ cross ion carbon (with TE) to the sum of free nucleon cross sections as a function of $Q^{2}$ for neutrino and antineutrino energies above $3 \mathrm{GeV}$

The top panel of Fig. 15 shows experimental values of $\mathcal{R}_{T}$ as a function of $Q^{2}$. The black points are extracted from Carlson et al [28], and the higher $Q^{2}$ are re-extracted from $\mathrm{QE}$ data from the JUPITER collaboration [29]. The $Q^{2}$ dependence of $\mathcal{R}_{T}$ is parametrized by the expression:

$\mathcal{R}_{T}=1+A Q^{2} e^{-Q^{2} / B}$ with $\mathrm{A}=5.194$ and $\mathrm{B}=0.376 \mathrm{GeV}^{2}$. The electron scattering data indicate that the transverse enhancement is maximal near $Q^{2}=0.3 \mathrm{GeV}^{2}$ and is small for $Q^{2}$ greater than $1.5 \mathrm{GeV}^{2}$ This parametrization is valid for carbon $(A=12)$ (it is also an approximate representation for higher A nuclei).

We assume that the enhancement in the transverse $\mathrm{QE}$ cross section can be described by a modification of $\mathcal{G}_{M}^{V}\left(Q^{2}\right)=$ $G_{M p}\left(Q^{2}\right)-G_{M n}\left(Q^{2}\right)$ for nucleons bound in carbon, where $G_{M p}$ and $G_{M n}$ are the magnetic form factor of the proton and neutron, respectively (as measured in electron scattering experiments). We use the parametrization of $\mathcal{R}_{T}$ to modify $G_{M p}$ and $G_{M n}$ for bound nucleons as follows.

$$
\begin{aligned}
& G_{M p}^{\text {nuclear }}\left(Q^{2}\right)=G_{M p}\left(Q^{2}\right) \times \sqrt{1+A Q^{2} e^{-Q^{2} / B}} \\
& G_{M n}^{\text {nuclear }}\left(Q^{2}\right)=G_{M n}\left(Q^{2}\right) \times \sqrt{1+A Q^{2} e^{-Q^{2} / B}} .
\end{aligned}
$$

Transverse enhancement increases the overall neutrino and antineutrino cross sections and changes the shape of the differential cross section as a function of $Q^{2}$ as shown in the bottom panel of Fig. 15.

Figure 16 shows the neutrino and antineutrino QE cross sections on ${ }^{12} \mathrm{C}$ with $\mathrm{TE}$ and without $\mathrm{TE}$ as a function of neutrino energy. The cross section for neutrinos is shown on the top panel and the cross section for antineutrinos is shown in bottom panel. Figure 17 shows the ratio of the neutrino and antineutrino $\mathrm{QE}$ cross sections on ${ }^{12} \mathrm{C}$ with $\mathrm{TE}$ to the sum of free nucleon cross sections as a function of energy. The ratio for neutrinos is shown in the top panel and the ratio for antineutrinos is shown inn the bottom panel. On average the overall cross section is increased by about $18 \%$.

Note that TE is a $2 \mathrm{p} 2 \mathrm{~h}$ process. Therefore, when TE is included in the model prediction, the relative fractions of the $1 \mathrm{p} 1 \mathrm{~h}$ and $2 \mathrm{p} 2 \mathrm{~h}$ should be changed as follows: 

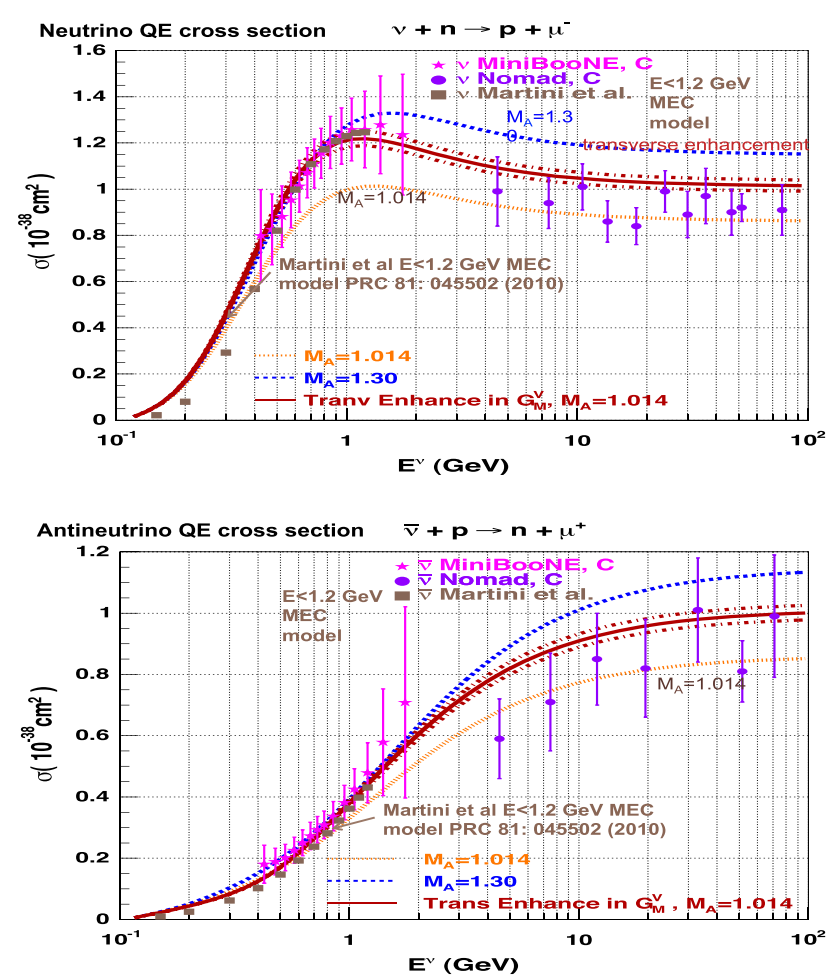

Fig. 16 The neutrino QE cross section on carbon with TE and without $\mathrm{TE}$ as a function of neutrino energy. The cross section for neutrinos is shown on the top panel and the cross section for antineutrinos is shown in bottom panel

$f_{1 p 1 h}^{(\text {with TE) }}=\frac{f_{1 p 1 h}}{1.18}$

$f_{2 p 2 h}^{(\text {with TE) }}=\frac{f_{2 p 2 h}+0.18}{1.18}$

In the above prescription, the energy sharing between the two nucleons in the final state for the $2 \mathrm{p} 2 \mathrm{~h}$ TE process is the same as for the $2 \mathrm{p} 2 \mathrm{~h}$ process from short range two nucleon correlations. We can make other assumptions about the energy sharing between the two nucleus for the TE process. For example one can chose to use a uniform angular distribution of the two nucleons in the center of mass of the two nucleons as is done in NuWro [6,7]. This can easily be done in a neutrino $\mathrm{MC}$ event generator, since once the events are generated, one can add an additional step and change the energy sharing between the two nucleons.

In summary, we extract the TE contribution by taking the difference between electron scattering data and the predictions of the $\psi^{\prime}$ formalism for QE scattering. Therefore, predictions using ESF for QE with the inclusion of the TE contribution fully describe electron scattering data by construction.

Including the TE model in neutrino Monte Carlo generators is relatively simple. The first step is to modify the magnetic form factors for the proton and neutron as given in Eq. 10. This accounts for the increase in the integrated QE cross section. The second step is to change the relative faction of
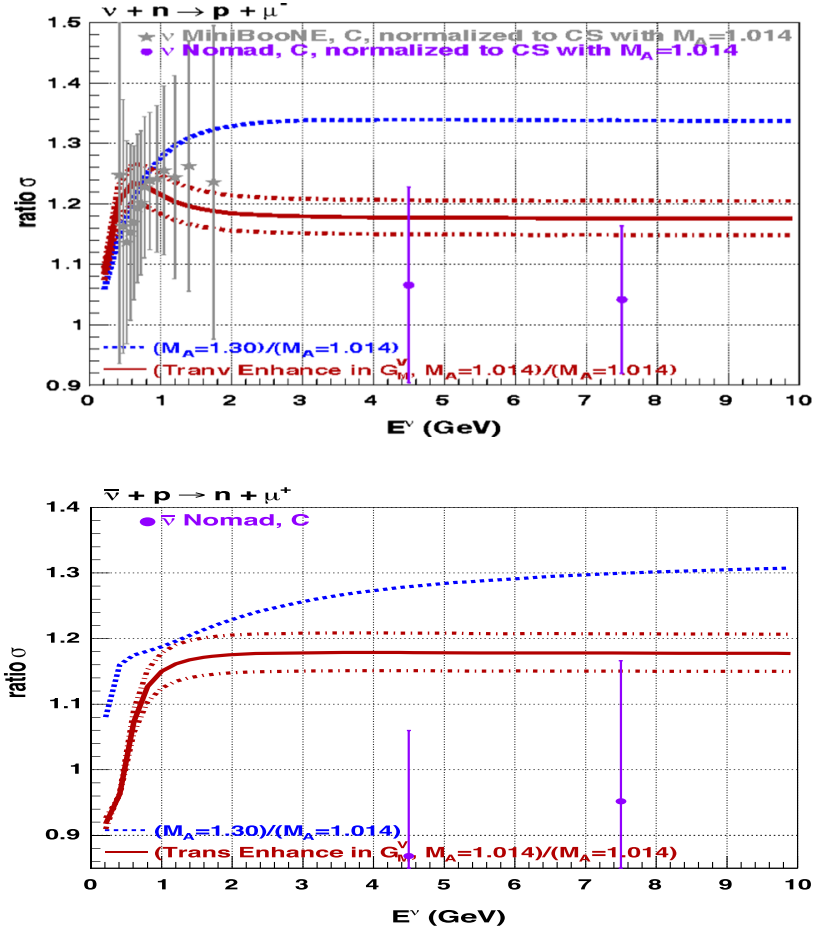

Fig. 17 The ratio of the total neutrino QE cross section on carbon with TE to sum of free nucleon cross sections as a function of energy. The ratio for neutrinos is shown on the top panel and the ratio for antineutrinos is shown in bottom panel. On average the overall cross section is increased by about $18 \%$

the $1 \mathrm{p} 1 \mathrm{~h}$ and $2 \mathrm{p} 2 \mathrm{~h}$ process as given in Eq. 11, which changes shape of the QE distribution in $v$.

The effective spectral function model and the TE model are not coupled. One can use the effective spectral function to describe the scattering from independent nucleons, and use another theoretical model to account for the additional contribution from multi nucleon process. Alternatively, one can use an alternative model for the scattering from independent nucleons and use the TE model to account for the additional contribution from multi nucleon processes.

\section{Effective spectral functions for deuterium}

Neutrino charged current QE cross sections for deuterium are not modeled in current neutrino Monte Carlo generators. We find that neutrino interactions on deuterium can also be modeled with an effective spectral function.

We use the theoretical calculations of reference [20] to predict the shape of the transverse differential cross section $\left(\frac{1}{\sigma} \frac{d \sigma}{d v}\left(Q^{2}, v\right)\right)$ for deuterium at several values of $Q^{2}$ as a function of $\Delta v=v-Q^{2} / 2 M$. These theoretical calculations are in agreement with electron scattering data. We tune the parameters of the effective spectral function to reproduce the spectra predicted by the theoretical calculations of reference [20]. 


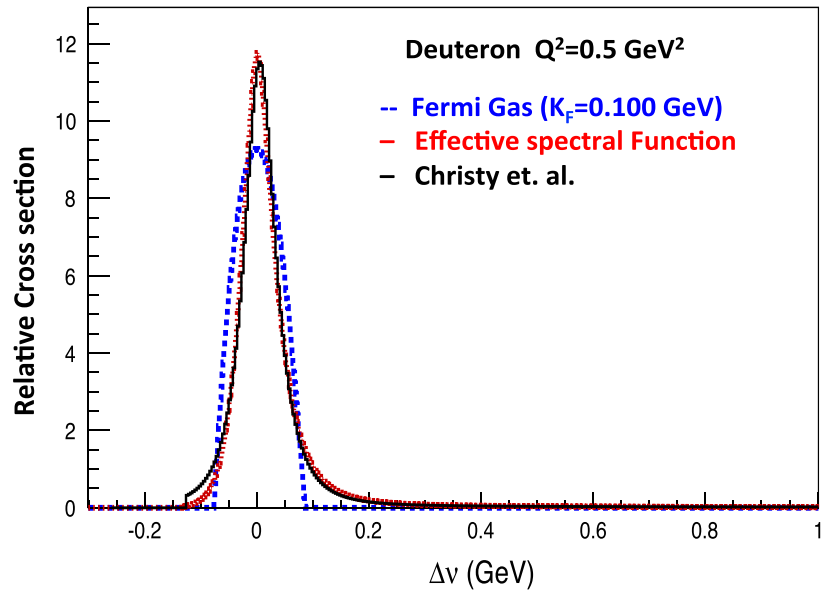

Fig. 18 Comparisons of model predictions for $\frac{1}{\sigma} \frac{d \sigma}{d v}\left(Q^{2}, v\right)$ as a function of $\Delta v=v-Q^{2} / 2 M$ for QE electron scattering on the deuterium at $Q^{2}=0.5 \mathrm{GeV}^{2}$. The solid black line is the prediction from reference [20] (which agrees with electron scattering data). The red line is the prediction of the best fit parameters for effective spectral function the deuteron. The blue line is the prediction for a Fermi gas with a Fermi momentum $K_{F}=0.100 \mathrm{GeV}$. The predictions with the effective spectral function are in agreement with the calculations of reference [20]

Figure 18 shows comparisons of model predictions for $\frac{1}{\sigma} \frac{d \sigma}{d v}\left(Q^{2}, \nu\right)$ for $\mathrm{QE}$ electron scattering on deuterium as a function of $\Delta v=v-Q^{2} / 2 M$ at $Q^{2}=0.5 \mathrm{GeV}^{2}$. The solid black line is the prediction from reference [20]. The red line is the prediction of the best fit parameters for the effective spectral function for deuterium. For comparison we also show (in blue) the prediction for a Fermi gas model with Fermi momentum $K_{F}=0.100 \mathrm{GeV}$. A comparison between the momentum distribution for the effective spectral function for deuterium and the momentum distribution for a Fermi gas with $K_{F}=0.100 \mathrm{GeV}$ is shown in Fig. 19

For reference, we note that the current default version of GENIE cannot be used nuclei with atomic weight $A<7$. This is because GENIE uses $K_{F}=0.169 \mathrm{GeV}$ (which has been extracted by Moniz [9-11] for ${ }^{3} \mathrm{Li}_{6}$ ) for all nuclei which have atomic weight $A<7$. GENIE with the implementation of the effective spectral function can be used for all nuclei. Recently, the effective spectral function has been implemented as an option in private versions of NEUT [30] and GENIE [31].

The best fit parameters for the effective spectral function for deuterium are given in Table 2. For deuterium, the $2 \mathrm{p} 2 \mathrm{~h}$ process is the only process that can happen .

\section{Pauli suppression}

The Pauli suppression in deuterium is smaller than the Pauli suppression in heavier nuclei. The multiplicative Pauli suppression factor for charged current neutrino scattering on deuterium $K_{\text {Pauli }}^{\text {deuter }}$ has been calculated by S. K. Singh $[21,22] . K_{\text {Pauli }}^{\text {deer }}$ can be parametrized $[23,24]$ by the fol-

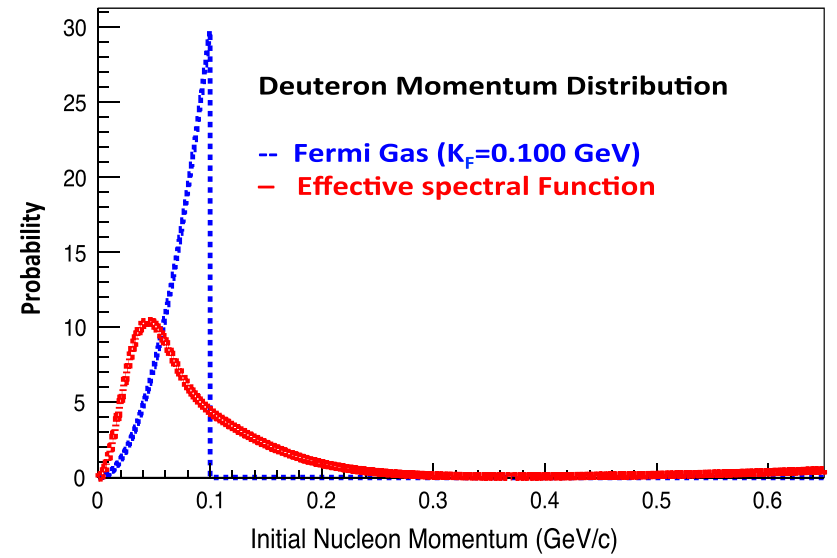

Fig. 19 The momentum distribution for the effective spectral function for deuterium (shown in red). Also shown (in blue) is the momentum distribution for a Fermi gas with $K_{F}=0.10 \mathrm{GeV}$. Note that we set the probability to zero for $k>K_{M}$ where $K_{M}=0.65 \mathrm{GeV}$

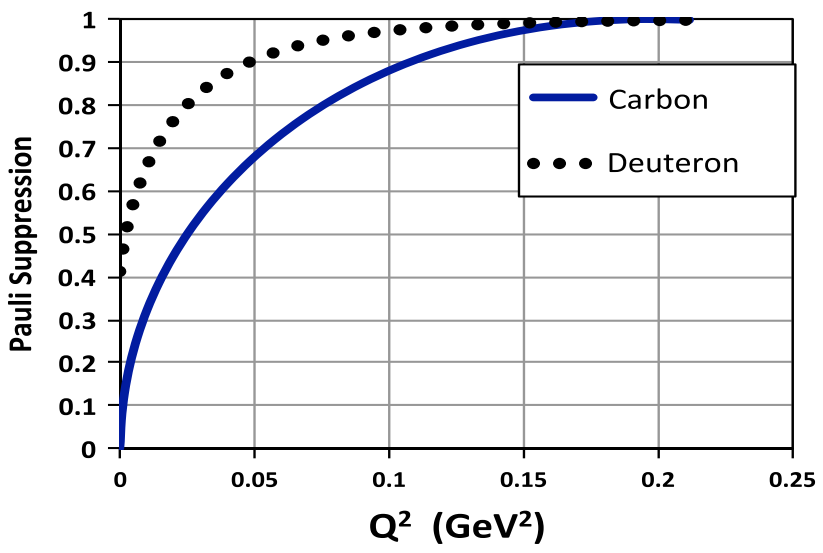

Fig. 20 A comparison of the Pauli suppression factor $K_{\text {Pauli }}$ for carbon and the deuteron as a function of the square of the four momentum transfers $Q^{2}$. For the carbon factor (eq. 4) we assumed $|\mathbf{q}|=\sqrt{Q^{2}\left(1+Q^{2} /\left(4 M_{p}^{2}\right)\right)}$

lowing function.

$K_{\text {Pauli }}^{\text {deuterium }}=1-A e^{B\left(Q^{2}\right)^{C}}$

where, $\mathrm{A}=0.588918, \mathrm{~B}=-17.2306, \mathrm{C}=0.749157$. A comparison of the Pauli suppression factors for ${ }^{12} \mathrm{C}$ (Eq. 4) and deuterium (Eq. 12) is shown in Fig. 20. Note that Pauli suppression in deuterium occurs only in neutrino charged current processes (because there are two protons in the final state). There is no suppression for electron scattering or neutrino neutral current processes.

\section{Conclusion}

We present parameters for an effective spectral function that reproduce the prediction for $\frac{1}{\sigma} \frac{d \sigma}{d v}\left(Q^{2}, v\right)$ from the best currently available models for charged current $\mathrm{QE}$ scattering on 
nuclear targets. We present parameters for a large number of nuclear targets from deuterium to lead.

Since most of the currently available neutrino MC event generators model neutrino scattering in terms of spectral functions, the effective spectral function can easily be implemented. For example, it has taken only a few days to implement the effective spectral function as an option in recent private versions of NEUT [30] and GENIE [31].

The predictions using ESF for QE with the inclusion of the TE contribution fully describe electron scattering data by construction.

Acknowledgments We thank Tomasz Golan for providing us with the predictions of NuWro, and Callum D. Wilkinson for implementation of the effective spectral function in NEUT.

Open Access This article is distributed under the terms of the Creative Commons Attribution License which permits any use, distribution, and reproduction in any medium, provided the original author(s) and the source are credited.

Funded by $\mathrm{SCOAP}^{3}$ / License Version CC BY 4.0.

\section{Appendix A: Fermi smearing in the resonance region}

The method of Bosted and Mamyan

Bosted and Mamyan [15] model Fermi motion effects for electron scattering data in the resonance and deep inelastic region by smearing the structure function $W_{1}\left(W^{\prime}, Q^{2}\right)$ on free nucleons to obtain the Fermi smeared structure function $W_{1}^{F}\left(W, Q^{2}\right.$ ). The smearing is done over $\left(W^{\prime}\right)^{2}$ (which is the square of the mass of the hadronic final state) at fixed values of $Q^{2}$. Bosted and Mamyan [15] use the following prescription.

$$
\begin{aligned}
W_{1}^{F}\left(W^{2}, Q^{2}\right) & =\sum_{i=1 \text { to } 99}\left[Z W_{1}^{p}\left(\left(W_{i}^{\prime}\right)^{2}, Q^{2}\right)\right. \\
& \left.+(A-Z) W_{1}^{n}\left(\left(W_{i}^{\prime}\right)^{2}, Q^{2}\right)\right] f_{i}\left(\xi_{i}\right)
\end{aligned}
$$

where the sum approximates an integral. Here, $W_{1}^{p}$ and $W_{1}^{n}$ are the free proton [26] and neutron [25] structure functions.

The shifted values $\left(W_{i}^{\prime}\right)^{2}$ are defined as

$$
\left(W_{i}^{\prime}\right)^{2}=W^{2}+\xi_{i} K_{F}|\mathbf{q}|-2 E_{\text {shift }}(v+M)
$$

where $\xi=2 k_{z} / K_{F}$, and $E_{\text {shift }}$ is the energy shift parameter. In the sum they use 99 values of $\xi_{i}$

$\xi_{i}=-3+6(i-1) / 98$

In the above equation $f_{i}(\xi)$ is the normalized probability for a nucleon to have a fractional longitudinal momentum $\xi=$ $2 k_{z} / K_{F}$. Bosted and Mamyan use the following normalized Gaussian for the probability.

$$
P^{B M}\left(k_{z}\right)=P^{B M}(\xi)=\mathcal{N}\left(\xi=0, \sigma_{\xi}=1\right)
$$
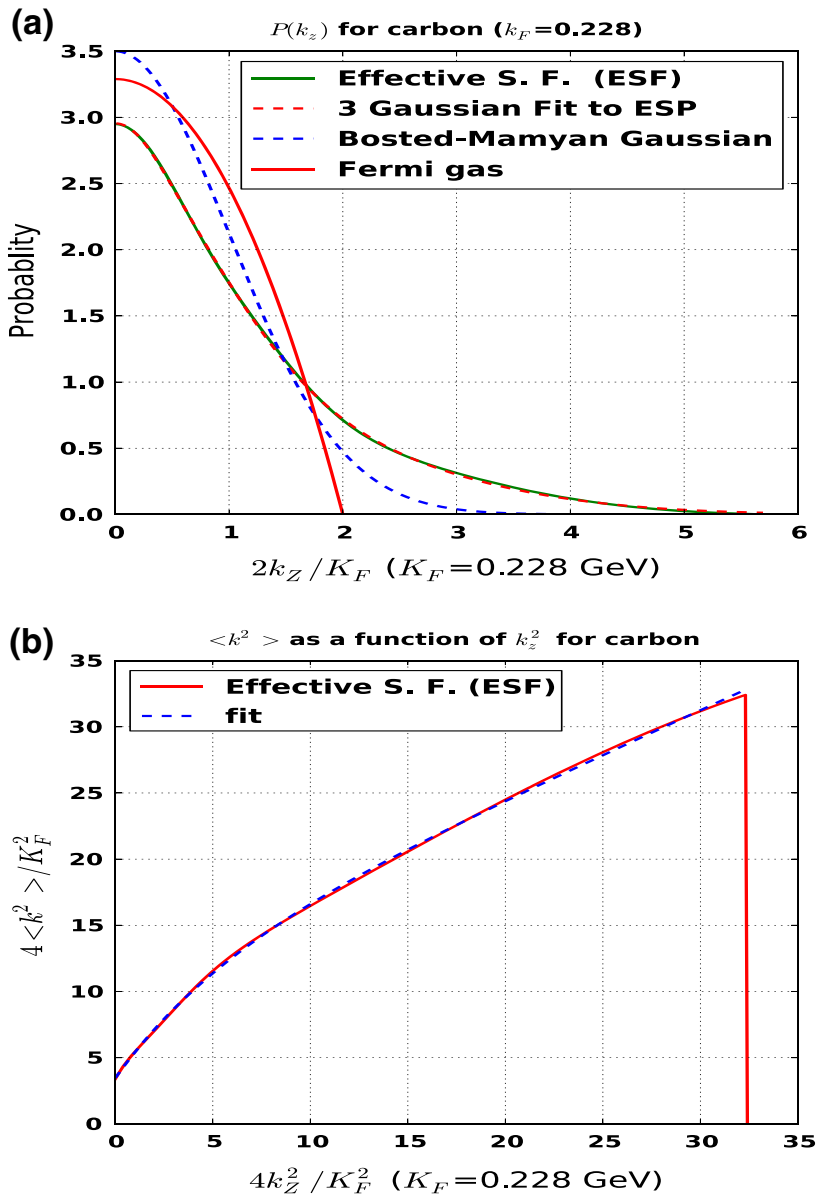

Fig. 21 (a) A comparison of the probability distribution $P\left(k_{z}\right)$ for ${ }^{12} \mathrm{C}$ plotted versus the variable $\xi=2 k_{z} / K_{F}$ for several spectral functions. (b) The average value of the square of nucleon moment $k^{2}$ for the ESF for ${ }^{12} \mathrm{C}$ versus the square of its $z$ component, $k_{z}^{2}$, shown in the form of $4<k^{2}>/ K_{F}^{2}$ versus $\xi^{2}=4 k_{z}^{2} / K_{F}^{2}$

which is equivalent to

$f_{i}=0.0245 e^{\left(-\xi_{i}^{2} / 2\right)}$

The sum is a step-wise integration over a Gaussian whose width is controlled by a Fermi momentum $K_{F}$, truncated at $\pm 3 \sigma\left(\xi_{i}\right.$ ranges from -3 to +3$)$, with a shift in central $W$ related to the energy shift parameter $E_{\text {shift. }}$. The values of $K_{F}$ and $E_{\text {shift }}$ used for the different nuclei are given in Table 1.

Fermi smearing in the resonance region using the effective spectral function

We have calculated the probability $P\left(k_{z}\right)$ for the effective spectral function $\left(P^{E S F}\left(k_{z}\right)\right)$ for ${ }^{12} \mathrm{C}$. The top panel of Fig. 21 shows the probability distributions $P\left(k_{z}\right)$ plotted versus the variable $\xi=2 k_{z} / K_{F}$ for the effective spectral function as compared to the distribution used by Bosted and Mamyan. Also shown is the probability distribution for the 
Table 4 Top half: Parameters for the parameterization of the one dimensional projection along $k_{z}$ of the effective spectral function $\left(P^{E S F}(\xi)\right)$ for various nuclei. The parameters for deuterium are shown in the col- umn labeled ${ }^{2} \mathrm{H}$. Bottom half: Parameters for the parameterization of the the mean $<k^{2}>$ as a function of $k_{z}$

\begin{tabular}{llllllllll}
\hline Parameter & ${ }^{2} \mathrm{H}$ & ${ }^{3} \mathrm{He}$ & ${ }^{4} \mathrm{He}$ & ${ }^{12} \mathrm{C}$ & ${ }^{20} \mathrm{Ne}$ & ${ }^{27} \mathrm{Al}$ & ${ }^{40} \mathrm{Ar}$ & ${ }^{56} \mathrm{Fe}$ & ${ }^{208} \mathrm{~Pb}$ \\
\hline$\Delta(\mathrm{MeV})$ & 0.13 & 5.3 & 14.0 & 12.5 & 16.6 & 12.5 & 20.6 & 15.1 \\
$f_{1 p 1 h}$ & 0 & 0.312 & 0.791 & 0.808 & 0.765 & 0.774 & 0.809 & 0.822 & 0.896 \\
$g_{1}$ & 0.2181 & 0.2560 & 0.1325 & 0.03819 & 0.1063 & 0.1063 & 0.1019 & 0.07645 & 0.1474 \\
$g_{2}$ & 0.6402 & 0.4343 & 0.3818 & 0.4168 & 0.3443 & 0.3443 & 0.3529 & 0.3892 & 0.3366 \\
$\sigma_{1}$ & 0.4465 & 0.7321 & 0.5724 & 0.3688 & 0.5538 & 0.5538 & 0.5401 & 0.5049 \\
$\sigma_{2}$ & 0.9920 & 1.459 & 1.054 & 0.910 & 0.9555 & 1.008 & 0.9416 & 0.9356 & 0.97962 \\
$\sigma_{3}$ & 3.233 & 3.126 & 2.122 & 1.928 & 1.870 & 1.935 & 1.873 & 1.889 \\
$K_{F}(\mathrm{GeV})$ & 0.100 & 0.115 & 0.190 & 0.228 & 0.230 & 0.236 & 0.241 & 0.241 \\
$a$ & 97.79 & 27.87 & 015.07 & 11.02 & 15.18 & 14.18 & 13.50 & 14.14 \\
$b$ & -0.2351 & 0.8118 & 0.6972 & 0.6772 & 0.5387 & 0.5466 & 0.5471 & 0.5252 \\
$c$ & 96.24 & 22.53 & 11.18 & 7.770 & 11.66 & 10.74 & 10.15 & 10.73 \\
$d$ & 43.34 & 15.54 & 8.692 & 5.441 & 8.482 & 8.001 & 7.169 & 7.536 \\
\hline
\end{tabular}

Fermi Gas model with $K_{F}=0.228$. The effective spectral function extends to higher momentum. In order to implement the effective spectral function we have fit $P^{E S F}\left(k_{z}\right)$ to a sum of three normal Gaussians with zero mean and different standard deviations $\sigma_{\xi}=\sigma_{i}$, and fractions $g_{1}, g_{2}$, and $g_{3}=1-g_{1}-g_{2}$.

$$
\begin{aligned}
P^{E S F}(\xi) & =g_{1} \mathcal{N}\left(\sigma_{1}\right)+g_{2} \mathcal{N}\left(\sigma_{2}\right)+g_{3} \mathcal{N}\left(\sigma_{3}\right) \\
\mathcal{N}\left(\sigma_{j}\right) & =\frac{1}{\sigma_{j} \sqrt{2 \pi}} e^{-0.5 \xi^{2} / \sigma_{j}^{2}}
\end{aligned}
$$

where $g_{1}=0.0382, g_{2}=0.417, \sigma_{1}=0.369, \sigma_{2}=0.910$, and $\sigma_{3}=1.928$. For smearing with the $\xi$ distribution of the effective spectral function we also use a 99 step integration in $\xi_{i}$ where,

$$
\begin{aligned}
\xi_{i} & =-6+12(i-1) / 98 \\
f_{i}(\xi) & =\frac{g_{1}}{8.1585} \mathcal{N}\left(\sigma_{1}\right)+\frac{g_{2}}{8.1585} \mathcal{N}\left(\sigma_{2}\right)+\frac{g_{3}}{8.1585} \mathcal{N}\left(\sigma_{3}\right)
\end{aligned}
$$

Here, the sum is a step-wise integration over a Gaussian whose width is controlled by a Fermi momentum $K_{F}$, truncated at $\pm 6 \sigma$ ( $\xi_{i}$ ranges from -6 to +6 ), with a shift in central $W$ related to removal energy. The values of $K_{F}$ used for the different nuclei are given in Table 1.

Bosted and Mamyan calculate the shifted values of $W^{\prime}$ (Eq. 14) using a fixed value for the energy shift $E_{\text {shift }}$. Instead, we calculate the shifted values of $W^{\prime}$ (Eq. 21) using the offshell neutron energies $\left(E_{n}\right)$ for the $1 \mathrm{p} 1 \mathrm{~h}$ (Eq. 22) and $2 \mathrm{p} 2 \mathrm{~h}$ (Eq. 22) processes, respectively. In order calculate the $k$ dependent off-shell neutrino energies we need to find the average $k^{2}$ as a function of $\xi$.

The bottom panel of Fig. 21 shows the average value of the square of nucleon moment $<k^{2}>$ versus the square of its $z$ component $\left(k_{z}^{2}\right)$ calculated for the effective spectral function for ${ }^{12} \mathrm{C}$. What is shown specifically is $4<k^{2}>/ K_{F}^{2}$ as a function of $\xi^{2}=4 k_{z}^{2} / K_{F}^{2}$. We parameterize $<k^{2}>$ by the following function:

$$
<k^{2}\left(\xi^{2}\right)>=\frac{K_{F}^{2}}{4}\left(a+b \xi^{2}-c e^{-\xi^{2} / d}\right)
$$

We repeat the analysis for all other nuclei. The top panel of Fig. 22 shows $P^{E S F}\left(\xi=2 K_{z} / K_{F}\right)$ for light nuclei. The middle panel shows $\left.P^{E S F}(\xi)\right)$ for heavy nuclei, and the bottom panel shows the mean $<k^{2}>$ as a function of $k_{Z}^{2}$ presented as as $4<k^{2}>/ K_{F}^{2}$ versus $\left(\xi^{2}=4 K_{z}^{2} / K_{F}^{2}\right)$.

The parameters for nuclei from ${ }^{2} \mathrm{H}$ to ${ }^{208} \mathrm{~Pb}$ are given in Table 4. However, we note that aside from ${ }^{2} \mathrm{H}$ (deuterium) and ${ }^{3} \mathrm{He}$, the functions for all nuclei are very similar. Therefore, as a good approximation, the parameters for ${ }^{12} \mathrm{C}$ can be used for all nuclei above $\mathrm{A}=12$, and also for ${ }^{4} \mathrm{He}$ (provided that the appropriate Fermi momentum for each nucleus as given in Table 4 is used for each nucleus).

Smearing with the effective spectral function requires that the shifted value of $W_{i}^{\prime}$ are different for the the $1 \mathrm{p} 1 \mathrm{~h}$ and $2 \mathrm{p} 2 \mathrm{~h}$ contributions.

$$
\begin{aligned}
W^{2} & =M_{n}^{2}+2 M_{n} v-Q^{2} \\
\left(M_{n}^{\prime}\right)^{2} & =\left(E_{n}\right)^{2}-V k^{2} \\
\left(W_{i}^{\prime}\right)^{2} & =\left(M_{n}^{\prime}\right)^{2}+2 E_{n} v-\xi_{i} K_{F}|\mathbf{q}|-Q^{2}
\end{aligned}
$$

Therefore,

$$
\begin{aligned}
\left(W_{i}^{\prime}\right)^{2} & =W^{2}-\xi_{i} K_{F}|\mathbf{q}|-V<k^{2}\left(\xi^{2}\right)> \\
& +\left[\left(E_{n}\right)^{2}-M_{n}^{2}\right]+2 v\left[E_{n}-M_{n}\right]
\end{aligned}
$$

Here $<k^{2}\left(\xi^{2}\right)>$ is given by Eq. 20 . 

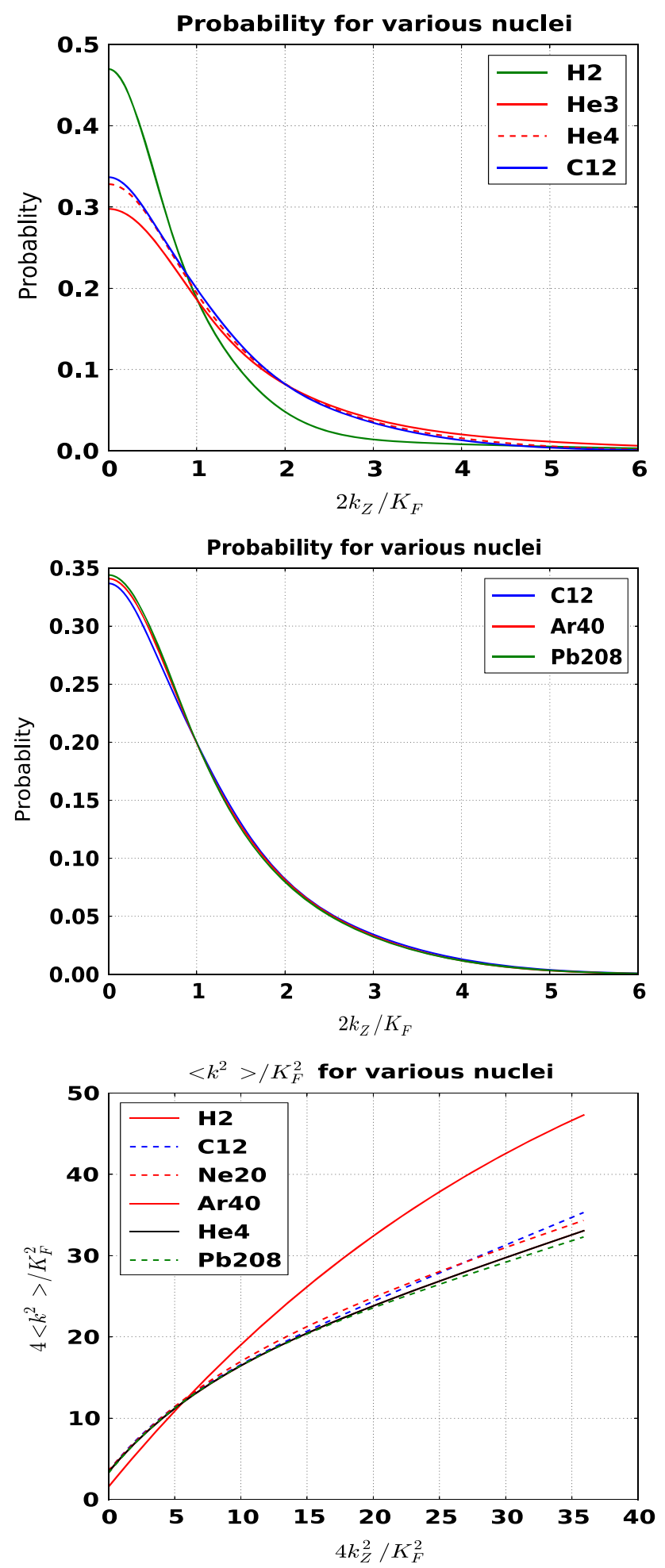

Fig. 22 Top panel: $P^{E S F}\left(\xi=2 K_{z} / K_{F}\right)$ for light nuclei. Middle panel: $P^{E S F}(\xi)$ for heavy nuclei. Bottom panel: The mean $\left\langle k^{2}>\right.$ as a function of $k_{Z}^{2}$ shown as $4<k^{2}>/ K_{F}^{2}$ versus $\left(\xi^{2}=4 K_{z}^{2} / K_{F}^{2}\right)$. Aside from deuterium (labeled H2) and Helium 3 (labeled He3) the functions for all nuclei are similar. Therefore, to a good approximation, the functions for ${ }^{12} \mathrm{C}$ can be used all nuclei above $\mathrm{A}=12$, and also for for Helium 4 (provided that the appropriate Fermi momentum for each nucleus as given in Table 4 is used for each nucleus)

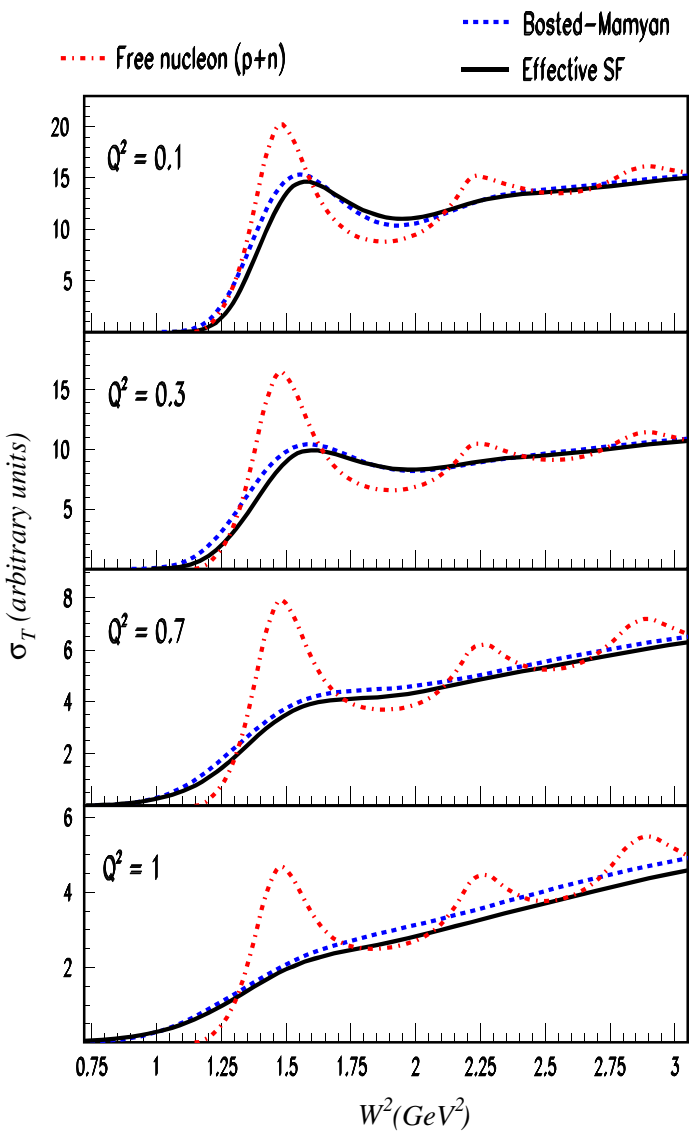

Fig. 23 The results of Fermi motion smearing in ${ }^{12} \mathrm{C}\left(K_{F}=0.2280\right.$ $\mathrm{GeV}$ ) of the free nucleon cross sections in the resonance region using the effective spectral function. The results are shown for several values of $Q^{2}$ and compared to the Fermi motion smearing used by Bosted and Mamyan

As mentioned earlier, the term $V\left(Q^{2}\right)$ multiplying $k^{2}$ in Eqs. 6, 8, 9, 21, 22, and 23 should be 1.0. However, we find that in order to make the spectral function agree with $\psi^{\prime}$ superscaling at very low $Q^{2}$ (e.g. $Q^{2}<0.3 \mathrm{GeV}^{2}$ ) we need to apply a $Q^{2}$-dependent correction of the form $V=1-e^{-x} Q^{2}$ where $x=12.04 \mathrm{GeV}^{-2}$. This term, shown in Fig. 5, accounts for the final state interaction (of the first kind) at low $Q^{2}$.

For the $1 \mathrm{p} 1 \mathrm{~h}$ process $E_{n}$ is given by Eq. 8, which when plugged into Eq. 21 for $\left(W_{i}^{\prime}\right)^{2}$ yields the following expression:

$$
\begin{aligned}
\left(W_{i}^{\prime}\right)_{1 p 1 h}^{2} & =W^{2}-\xi_{i} K_{F}|\mathbf{q}|-V<k^{2}\left(\xi^{2}\right)> \\
& -2[v+M]\left[\Delta+\frac{V<k^{2}\left(\xi^{2}\right)>}{2 M_{A-1}^{*}}\right]
\end{aligned}
$$

For the $2 \mathrm{p} 2 \mathrm{~h}$ process $E_{n}$ is given by Eq. 9 which when plugged into Eq. 21 for $\left(W_{i}^{\prime}\right)^{2}$ yields the following expression: 


$$
\begin{aligned}
& \left(W_{i}^{\prime}\right)_{2 p 2 h}^{2}=W^{2}-\xi_{i} K_{F}|\mathbf{q}|-V<k^{2}\left(\xi^{2}\right) \\
& >+\left[\left(\left(M_{p}+M_{n}\right)-2 \Delta-\sqrt{V<k^{2}\left(\xi^{2}\right)>+M_{p}^{2}}\right)^{2}-M_{n}^{2}\right] \\
& +2 v\left[\left(M_{p}+M_{n}\right)-2 \Delta-\sqrt{V<k^{2}\left(\xi^{2}\right)>+M_{p}^{2}}-M_{n}\right]
\end{aligned}
$$

When smearing the proton and neutron structure functions, the $1 \mathrm{p} 1 \mathrm{~h}$ and $2 \mathrm{p} 2 \mathrm{~h}$ processes are weighted by the relative fractions given in Table 4.

Figure 23 shows the results of Fermi motion smearing in ${ }^{12} \mathrm{C}\left(K_{F}=0.2280 \mathrm{GeV}\right)$ of the free nucleon cross sections in the resonance region using the effective spectral function. The results are shown for several values of $Q^{2}$ and compared to the Fermi motion smearing used by Bosted and Mamyan. The spectra smeared with the effective spectral function are are shifter to higher values of $W^{2}$.

\section{Appendix B: Calculation of the shape of the quasielastic peak}

We calculate the shape of the quasielastic peak $\frac{1}{\sigma} \frac{d \sigma}{d v}\left(Q^{2}, v\right)$ at fixed $Q^{2}$ using the expressions below. The on-shell elastic $W_{2}$ structure function for the scattering of neutrinos on free neutrons [27] is given by

$W_{2}^{\text {on-shell }}=G\left(Q^{2}\right) \delta\left(v-Q^{2} / 2 M\right)$

where $G\left(Q^{2}\right)$ is given in terms of vector and axial form factors.

$$
\begin{aligned}
G\left(Q^{2}\right) & =\left|\mathcal{F}_{V}\left(Q^{2}\right)\right|^{2}+\left|\mathcal{F}_{A}\left(Q^{2}\right)\right|^{2} \\
\left|\mathcal{F}_{V}\left(Q^{2}\right)\right|^{2} & =\frac{\left[\mathcal{G}_{E}^{V}\left(Q^{2}\right)\right]^{2}+\tau\left[\mathcal{G}_{M}^{V}\left(Q^{2}\right)\right]^{2}}{1+\tau} .
\end{aligned}
$$

From conserved vector current $(\mathrm{CVC}) \mathcal{G}_{E}^{V}\left(Q^{2}\right)$ and $\mathcal{G}_{M}^{V}\left(Q^{2}\right)$ are related to the electron scattering electromagnetic form factors [32] $G_{E}^{p}\left(Q^{2}\right), G_{E}^{n}\left(Q^{2}\right), G_{M}^{p}\left(Q^{2}\right)$, and $G_{M}^{n}\left(Q^{2}\right)$ :

$\mathcal{G}_{E}^{V}\left(Q^{2}\right)=G_{E}^{p}\left(Q^{2}\right)-G_{E}^{n}\left(Q^{2}\right)$,

$\mathcal{G}_{M}^{V}\left(Q^{2}\right)=G_{M}^{p}\left(Q^{2}\right)-G_{M}^{n}\left(Q^{2}\right)$.

The axial form factor $\mathcal{F}_{A}$ can be approximated by the dipole form

$\mathcal{F}_{A}\left(Q^{2}\right)=\frac{g_{A}}{\left(1+\frac{Q^{2}}{\mathcal{M}_{A}^{2}}\right)^{2}}$,

where $g_{A}=-1.267$, and $\mathcal{M}_{A}=1.014 \mathrm{GeV}$ [32]. Fits that include modifications to dipole form for both vector and axial form factors can be found in Ref. [32].

We note that when we calculate the shape of the QE peak $\frac{1}{\sigma} \frac{d \sigma}{d v}\left(Q^{2}, v\right)$ at fixed $Q^{2}$ the function $G\left(Q^{2}\right)$ cancels out.
For the scattering from an off-shell nucleon, the energy conservation $\delta$ function takes the following form:

$$
\begin{aligned}
M_{p}^{2} & =\left(M_{n}^{\prime}\right)^{2}+2 E_{n} v-2|q| k_{z}-Q^{2} \\
v & =\frac{Q^{2}+2|q| k_{z}+M_{p}^{2}-\left(M_{n}^{\prime}\right)^{2}}{2 E_{n}} \\
W_{2}^{\text {off }} & =G\left(Q^{2}\right) \delta\left(v-\frac{Q^{2}+2|q| k_{z}+M_{p}^{2}-\left(M_{n}^{\prime}\right)^{2}}{2 E_{n}}\right)
\end{aligned}
$$

where $k_{z}=k \cos \theta$. Here $\theta$ is the angle between the direction of the momentum transfer $\mathbf{q}$ and $\mathbf{k}$. The shape of the quasielastic peak $\frac{1}{\sigma} \frac{d \sigma}{d v}\left(Q^{2}, v\right)$ at fixed $Q^{2}$ is then given by

$\frac{d \sigma}{d \nu}\left(Q^{2}, \nu\right) \propto \int_{0}^{K_{M}} 2 \pi \int_{-1}^{1} W_{2}^{o f f}|\phi(k)|^{2} k^{2} d \cos \theta d k$

where $P(k)=|\phi(k)|^{2} 4 \pi k^{2}$ is the probability distribution for a nucleon to have a momentum $k=|\mathbf{k}|$ in the nucleus. For all of the momentum distributions that we investigate we set the probability to zero for $k>K_{M}$ where $K_{M}=0.65 \mathrm{GeV}$.

The shape of the quasielastic peak for the Fermi gas model

For the Fermi gas model we can get an approximate distribution for $\frac{d \sigma}{d \nu}\left(Q^{2}\right)$ in closed form. We use this calculation as a check on our results which are obtained using Eq. 28.

For the Fermi gas model we do the calculation in cylindrical coordinate

$\left(2 \pi k^{2} d \cos \theta d k=\pi d k_{r}^{2} d k_{z}\right)$

$k=\sqrt{k_{r}^{2}+k_{z}^{2}}$.

Equation 28 can then be written as

$$
\begin{aligned}
W_{2}^{o f f} & \propto \frac{E_{n}}{|\mathbf{q}|} \delta\left(k_{z}-\frac{1}{2|\mathbf{q}|}\left(2 E_{n} \nu-Q^{2}-M_{p}^{2}+\left(M_{n}^{\prime}\right)^{2}\right)\right. \\
\frac{d \sigma}{d \nu}\left(Q^{2}\right) & \propto \int_{-K_{F}}^{K_{F}} \int_{0}^{K_{F}-k_{z}^{2}} W_{2}^{o f f}|\phi(k)|^{2} \pi d k_{r}^{2} d k_{z}
\end{aligned}
$$

For the Fermi gas model the momentum distribution is zero for $k>K_{F}$, and for $k<K_{F}$ it is given by

$$
\begin{aligned}
|\phi(k)|^{2} & =\frac{1}{N} \quad N=\frac{4}{3} \pi K_{F}^{3} \\
P(k) d k & =|\phi(k)|^{2} 4 \pi k^{2} d k=\frac{1}{N} 4 \pi k^{2} d k
\end{aligned}
$$

For simplicity, we assume that the energy of the off shell neutron is a constant which is independent of $\mathrm{k}$. Using < 


$$
\begin{aligned}
k^{2}>=\frac{3}{5} K_{F}^{2} & \text { we obtain } \\
<E_{n}> & =M_{n}-\Delta-\frac{3 K_{F}^{2}}{10 M_{A-1}^{*}} \\
<\left(M_{n}^{\prime}\right)^{2}> & =<E_{n}>^{2}-3 K_{F}^{2} / 5 \\
\frac{d \sigma}{d \nu}\left(Q^{2}\right) & \propto \int_{-K_{F}}^{K_{F}} \int_{0}^{K_{F}-k_{z}^{2}} W_{2}^{o f f}|\phi(k)|^{2} \pi d k_{r}^{2} d k_{z} \\
& =\int_{-K_{F}}^{K_{F}} W_{2}^{o f f} \frac{3}{4 K_{F}^{3}}\left(K_{F}^{2}-k_{z}^{2}\right) d k_{z}
\end{aligned}
$$

Integrating the $\delta$ function in Eq. 27 over $k_{z}$ we get

$$
\begin{array}{r}
k_{z}=\frac{1}{2|\mathbf{q}|}\left[2<E_{n}>v-Q^{2}-M_{p}^{2}+<\left(M_{n}^{\prime}\right)^{2}>\right] \\
\frac{1}{\sigma} \frac{d \sigma}{d v}\left(Q^{2}, v\right)=\frac{<E_{n}>}{|\mathbf{q}|} \frac{3}{4} \frac{\left(K_{F}^{2}-k_{z}^{2}\right)}{K_{F}^{3}} .
\end{array}
$$

The above equation satisfies the normalization condition

$$
\int \frac{<E_{n}>3}{|\mathbf{q}|} \frac{3}{4} \frac{\left(K_{F}^{2}-k_{z}^{2}\right)}{K_{F}^{3}} d v=1
$$

\section{References}

1. C. Andreopoulos [GENIE Collaboration], Acta Phys. Pol. B 40, 2461 (2009)

2. C. Andreopoulos (GENIE), Nucl. Instrum. Methods A 614, 87 (2010)

3. H. Gallagher (NEUGEN), Nucl. Phys. Proc. Suppl. 112, 188 (2002)

4. Y. Hayato (NEUT), Nucl. Phys. Proc. Suppl. 112, 171 (2002)

5. D. Casper (NUANCE), Nucl. Phys. Proc. Suppl. 112, 171 (2002). http://nuint.ps.uci.edu/nuance/

6. J. Sobczyk, NuWro, PoS NUFACT 08, 141 (2008)

7. C. Juszczak, Acta Phys. Pol. B 40, 2507 (2009). http://borg.ift.uni. wroc.pl/nuwro/
8. T. Leitner, O. Buss, L. Alvarez-Ruso, GiBUU, Phys. Rev. C79, 034601 (2009). arXiv:0812.0587

9. E.J. Moniz et al., Phys. Rev. Lett. 26, 445 (1971)

10. E.J. Moniz, Phys. Rev. 184, 1154 (1969)

11. R.A. Smith, E.J. Moniz, Nucl. Phys. B43, 605 (1972)

12. O. Benhar, S. Fantoni, G. Lykasov, Eur. Phys. J. A 7(3), 415 (2000)

13. O. Benhar et al., Phys. Rev. C55, 244 (1997)

14. J.E. Amaro, M.B. Barbaro, J.A. Caballero, T.W. Donnelly, A. Molinari, I. Sick, Phys. Rev. C 71, 015501 (2005). arXiv:nucl-th/0409078

15. P.E. Bosted, V. Mamyan. arXiv: 1203.2262

16. M.E. Christy, Private communication (2014)

17. A. Bodek, J.L. Ritchie, Phys. Rev. D23, 1070 (1980)

18. A. Bodek, J.L. Ritchie, Phys. Rev. D24, 1400 (1981)

19. S. Mishra (NOMAD Collaboration), Private communication, fit parameters from E. Iacopini (1997)

20. M.E. Christy, N. Kalantarians, J.J. Ethier, W. Melnitchouk. In preparation (2014)

21. S.K. Singh, Nucl. Phys. B36, 419 (1972)

22. K. Singh, H. Arenhove, Nucl. Phys. B36, 419 (1972)

23. H. Budd, A. Bodek, J. Arrington, Nucl. Phys. Proc. Suppl. 139, 90 (2005)

24. H. Budd, A. Bodek, J. Arrington, Z. Phys. A 324, 3476 (1986)

25. P.E. Bosted, M.E. Christy, Phys. Rev. C77, 065206 (2008). arXiv:0711.0159

26. M.E. Christy, P.E. Bosted, Phys. Rev. C81, 055213 (2010). arXiv:0712.3731

27. A. Bodek, H.S. Budd, M.E. Christy, Eur. Phys. J. C 71, 1726 (2011)

28. J. Carlson, J. Jourdan, R. Schiavilla, I. Sick, Phys. Rev. C65, 024002 (2002)

29. A. Bodek, C. Keppel, M.E. Christy (Spokespersons JUPITER Collaboration), Jefferson Lab Experiment E04-001

30. C.D. Wilkinson, Private communication (NEUT)

31. Brian Coopersmith, Private communication (GENIE)

32. A. Bodek, S. Avvakumov, R. Bradford, H. Budd, Eur. Phys. J. C 53, 349 (2008) 